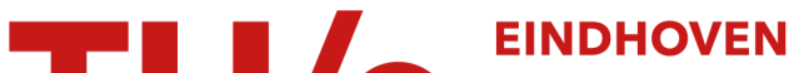 UNIVERSITY OF TECHNOLOGY
}

\section{Directional change of tracer trajectories in rotating Rayleigh- Bénard convection}

Citation for published version (APA):

Alards, K. M. J., Rajaei, H., Kunnen, R. P. J., Toschi, F., \& Clercx, H. J. H. (2018). Directional change of tracer trajectories in rotating Rayleigh-Bénard convection. Physical Review E, 97(6), [063105].

https://doi.org/10.1103/PhysRevE.97.063105

DOI:

10.1103/PhysRevE.97.063105

Document status and date:

Published: 18/06/2018

\section{Document Version:}

Publisher's PDF, also known as Version of Record (includes final page, issue and volume numbers)

\section{Please check the document version of this publication:}

- A submitted manuscript is the version of the article upon submission and before peer-review. There can be important differences between the submitted version and the official published version of record. People interested in the research are advised to contact the author for the final version of the publication, or visit the $\mathrm{DOI}$ to the publisher's website.

- The final author version and the galley proof are versions of the publication after peer review.

- The final published version features the final layout of the paper including the volume, issue and page numbers.

Link to publication

\section{General rights}

Copyright and moral rights for the publications made accessible in the public portal are retained by the authors and/or other copyright owners and it is a condition of accessing publications that users recognise and abide by the legal requirements associated with these rights.

- Users may download and print one copy of any publication from the public portal for the purpose of private study or research.

- You may not further distribute the material or use it for any profit-making activity or commercial gain

- You may freely distribute the URL identifying the publication in the public portal.

If the publication is distributed under the terms of Article 25fa of the Dutch Copyright Act, indicated by the "Taverne" license above, please follow below link for the End User Agreement:

www.tue.nl/taverne

Take down policy

If you believe that this document breaches copyright please contact us at:

openaccess@tue.nl

providing details and we will investigate your claim. 


\title{
Directional change of tracer trajectories in rotating Rayleigh-Bénard convection
}

\author{
Kim M. J. Alards, ${ }^{1, *}$ Hadi Rajaei, ${ }^{1}$ Rudie P. J. Kunnen, ${ }^{1}$ Federico Toschi, ${ }^{1,2,3}$ and Herman J. H. Clercx ${ }^{1}$ \\ ${ }^{1}$ Fluid Dynamics Laboratory, Department of Applied Physics, Eindhoven University of Technology, P.O. Box 513, \\ 5600 MB Eindhoven, The Netherlands \\ ${ }^{2}$ Centre of Analysis, Scientific Computing, and Applications W\&I, Department of Mathematics and Computer Science, Eindhoven University \\ of Technology, P.O. Box 513, 5600 MB Eindhoven, The Netherlands \\ ${ }^{3}$ Istituto per le Applicazioni del Calcolo, Consiglio Nazionale delle Ricerche, Via dei Taurini 19, 00185 Rome, Italy
}

(Received 8 March 2018; published 18 June 2018)

\begin{abstract}
The angle of directional change of tracer trajectories in rotating Rayleigh-Bénard convection is studied as a function of the time increment $\tau$ between two instants of time along the trajectories, both experimentally and with direct numerical simulations. Our aim is to explore the geometrical characterization of flow structures in turbulent convection in a wide range of timescales and how it is affected by background rotation. We find that probability density functions (PDFs) of the angle of directional change $\theta(t, \tau)$ show similar behavior as found in homogeneous isotropic turbulence, up to the timescale of the large-scale coherent flow structures. The scaling of the averaged (over particles and time) angle of directional change $\Theta(\tau)=\langle|\theta(t, \tau)|\rangle$ with $\tau$ shows a transition from the ballistic regime $\left[\Theta(\tau) \sim \tau^{c}\right.$ with $\left.c=1\right]$ for $\tau \lesssim \tau_{\eta}$, with $\tau_{\eta}$ the Kolmogorov timescale, to a scaling with smaller exponent $c$ for $\tau_{\eta} \lesssim \tau \lesssim T_{L}$, with $T_{L}$ the Lagrangian integral timescale. This scaling exponent is approximately constant in the weakly rotating regime (Rossby number Ro $\gtrsim 2.5$ ) and is decreasing for increasing rotation rates when Ro $\lesssim 2.5$. We show that this trend in the scaling exponent is related with the large-scale coherent structures in the flow; the large-scale circulation for Ro $\gtrsim 2.5$ and vertically aligned vortices emerging from the boundary layers (BLs) near the top and bottom plates and penetrating into the bulk for Ro $\lesssim 2.5$. In the viscous BLs, the PDFs of $\theta(t, \tau)$ and scaling properties of $\Theta(\tau)$ are in general different from those measured in the bulk and depend on the type of boundary layer, in particular whether the BL is of Prandtl-Blasius type (Ro $\gtrsim 2.5$ ) or of Ekman type (Ro $\lesssim 2.5$ ). When it is of Ekman type, a stronger dynamic coupling exists between the BL and the bulk of the flow, resulting in similar scaling exponents in BL and bulk.
\end{abstract}

DOI: 10.1103/PhysRevE.97.063105

\section{INTRODUCTION}

Rotating turbulent nonisothermal flows are relevant for many geophysical and astrophysical applications, such as ocean currents [1,2] and solar convection [3], as well as for industrial applications, such as convective cooling in turbomachinery [4,5]. In such turbulent flows, rotation changes the typical flow structures and their typical length and timescales [6,7]. We use a rotating Rayleigh-Bénard convection (RBC) setup, a fluid layer heated from below and cooled from above, where rotation is known to change the flow structures from a large-scale circulation (LSC) to vertically aligned vortical plumes [8-10]. Related to this transition, the boundary layer (BL) dynamics changes from a Prandtl-Blasius type for smaller rotation rates to the Ekman type for larger rotation rates [11].

The multiscale character of turbulence and the characteristics of the large-scale coherent flow structures can be studied by considering the geometry of tracer trajectories. Instantaneous measures for this geometry are the curvature and torsion of trajectories. Such curvature and torsion measurements of tracer trajectories in rotating RBC have shown that the predictions for the scaling of curvature and torsion probability density functions (PDFs) derived for homogeneous

\footnotetext{
*k.m.j.alards@tue.nl
}

isotropic turbulence (HIT) [12-15] are recovered, as long as measurements are performed in the turbulent bulk [16]. In the BLs, the PDFs scale differently consistent with the type of $\mathrm{BL}$, which is the Prandtl-Blasius type in the regime dominated by the LSC and the Ekman type in the regime affected by vertically aligned vortices. However, curvature and torsion of trajectories are instantaneous measures and do not capture the variety of timescales typical for turbulent flows. Due to this multiscale dynamics, tracer trajectories in turbulent flows change direction at every timescale. In RBC, trajectories are additionally expected to be affected by the large-scale coherent flow structures at larger timescales. We therefore extend the previous study on instantaneous measurements of the geometry of tracer trajectories [16] to scale-dependent measurements of this geometry by computing the directional change of tracer trajectories in (rotating) RBC up to the timescale of the large-scale coherent flow structures.

In a previous study [17], the directional change of trajectories at different timescales has been measured in (threedimensional) HIT on a periodic domain. A typical scaling of the average angle of directional change with the time increment $\tau$ was found in two different regimes: (i) in the regime of timescales smaller than the Kolmogorov timescale a ballistic scaling, proportional to $\tau$, was identified and (ii) for timescales larger than the Kolmogorov timescale but smaller than the Lagrangian integral timescale an inertial range was identified 
where the scaling was predicted as $\tau^{1 / 2}$, using Kolmogorov phenomenology. In RBC, the geometrical statistics might additionally give information on the typical coherent flow structures, i.e., the LSC for rotation unaffected RBC and vertically aligned plumes for rotating RBC. BLs with yet other flow characteristics develop at the horizontal plates. In this study, we will investigate whether a signature of these different flow structures is visible in the multiscale geometrical statistics of tracer trajectories.

The study on geometrical statistics in HIT [17] was extended to inertial particles in [18] and to two-dimensional (2D) turbulence in [19], where 2D turbulence forced at large scales, was compared to 2D turbulence forced at small scales. Only in the latter case the energy spectrum, that is then characterized by an inverse energy cascade, recovers an inertial range according to Kolmogorov phenomenology, with a scaling of $k^{-5 / 3}$, with $k$ the forcing wave number. It was shown that the $\tau^{1 / 2}$ scaling for the average angle of directional change in the inertial range is only recovered in the case of small-scale forcing, indicating that the $\tau^{1 / 2}$ scaling is clearly a flow-dependent phenomenon. In [19] also the effect of confinement is investigated by comparing $2 \mathrm{D}$ turbulence on a periodic domain to $2 \mathrm{D}$ turbulence on a confined domain. The signature of confinement is visible in the long-time convergence of the average angle; in an unbounded domain, often represented by a periodic domain, the statistics of the angle is equidistributed at large timescales and the angle converges to $\pi / 2$, while in a confined domain it goes to $2 \pi / 3$. This is shown not to be a result of modified velocity properties at the walls, but rather of nonequidistributed angles when the separation of subsequent points along a trajectory becomes of the order of the domain size [19].

In this work, we first focus on the angle of directional change in nonrotating $\mathrm{RBC}$ in a cylindrical domain. This setup poses already many extra challenges compared to HIT on a periodic domain. First, the setup is bounded with radial and horizontal walls, resulting in an inhomogeneous and anisotropic flow [2022]. Although anisotropy and inhomogeneity were shown not to affect the instantaneous curvature and torsion statistics in the bulk of the RBC flow, it is not clear how they will affect the geometry of tracer trajectories at longer timescales. Statistics is moreover collected in finite measurement volumes in the RBC cell, possibly imposing confinement and influencing the convergence of the average angle at large timescales, like in the 2D confined turbulence setup in [19], as also discussed above. Also, the level of turbulence in the RBC setup studied here is moderate and no clear inertial Kolmogorov range can be identified in the energy spectrum [23]. Therefore, it is not clear a priori what scaling to expect for the average angle of directional change in the regime of time increments larger than the Kolmogorov timescale.

When including rotation, the typical flow structures in RBC change from an LSC to vertically aligned plumes. The level of coherence of these structures is different, where vertically aligned vortices have longer vertical coherence times than the LSC [24]. Therefore, rotation is expected to have an effect on the angle of directional change, especially at larger timescales. Also, the level of turbulence [11,24] and the energy spectrum [25-27] are influenced by rotation. Here, we want to investigate whether a signature of the transition between the rotation unaffected (LSC dominated) and weakly rotation affected regime can be identified in the scaling of the average angle of directional change with time increments at timescales larger than the Kolmogorov timescale.

The BL dynamics is shown to be of importance for understanding the transitions between the different rotational regimes in $\mathrm{RBC}[10,24,28,29]$. In [16] it was shown that the scaling of curvature and torsion PDFs was different in the BL compared to the bulk, especially in the regime of lower rotation rates where a strong mean horizontal flow is present in the BL. We will extend the bulk measurements with measurements in the $\mathrm{BL}$ region at different rotation rates, such that we can study not only the effect of the different coherent flow structures in the bulk, but also the effect of the different BL dynamics on the angle of directional change at different timescales.

First, in Sec. II, we explain how the angles of directional change are computed for tracer trajectories in turbulence and we derive the scaling laws expected in HIT, based on [17]. Experimental and numerical methods for studying (rotating) $\mathrm{RBC}$ are briefly explained in Sec. III. Results are discussed in $\mathrm{Sec}$. IV where angular statistics and the scaling of the average angle of directional change are shown as a function of time and for both nonrotating and rotating $\mathrm{RBC}$ and measurements inside the bulk are compared to measurements in the $\mathrm{BL}$ regions of the flows. A summary and conclusion are given in Sec. V.

\section{MULTISCALE ANGULAR STATISTICS}

The geometry of a three-dimensional (3D) curve in space is described by its curvature and torsion. Curvature and torsion statistics of particle trajectories in turbulent flows give useful information on the typical flow structures [12-14,16]. However, these are instantaneous measures, dominated by the small-scale structures of the flow and therefore do not capture the multiscale dynamics of a turbulent flow [17]. A multiscale measurement related to the geometry of trajectories is proposed in $[17,30]$ as the directional change of fluid tracer trajectories. Following the procedure proposed by Bos, Kadoch, and Schneider in [17], we first define the spatial increment of trajectories, sketched in Fig. 1(a), as

$$
\delta \mathbf{x}(t, \tau)=\mathbf{x}(t)-\mathbf{x}(t-\tau),
$$

where $\mathbf{x}(t)$ is the position of the tracer particle at time $t$ and $\tau$ is the time increment between subsequent positions. Next, we introduce the cosine of the angle between these subsequent points along trajectories as

$$
\cos \theta(t, \tau)=\frac{\delta \mathbf{x}(t, \tau) \delta \mathbf{x}(t+\tau, \tau)}{|\delta \mathbf{x}(t, \tau)||\delta \mathbf{x}(t+\tau, \tau)|} .
$$

The curvature, denoted by $\kappa$, is related to the angle $\theta(t, \tau)$ in the limit of $\tau \rightarrow 0$ as

$$
\kappa=\lim _{\tau \rightarrow 0} \frac{\theta(t, \tau)}{2 \tau|\mathbf{u}(t)|},
$$

with $\mathbf{u}(t)$ the velocity of the trajectory at time $t$.

In HIT, the average angle $\Theta(\tau)=\langle|\theta(t, \tau)|\rangle$, with $\langle\ldots\rangle$ the ensemble average over all tracer trajectories and over time $t$, displays a power-law scaling with $\tau$ in the limit of $\tau \ll T_{L}$, with $T_{L}$ the Lagrangian integral timescale of the flow [17]. Again, we follow the procedure suggested in [17], where $\theta$ is 


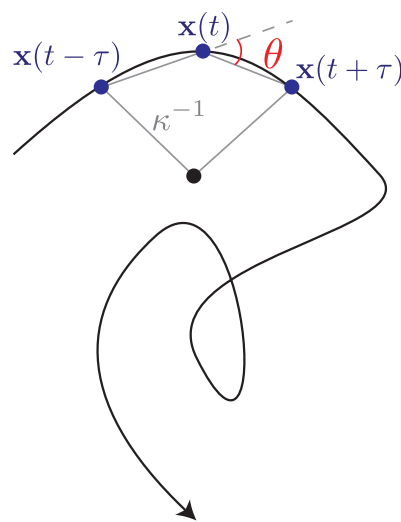

(a)

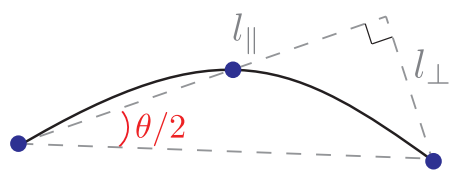

(b)
FIG. 1. (a) Sketch of the angle between subsequent time steps $\tau$ of the particle trajectory, where $\mathbf{x}(t)$ is the position of the particle at time $t$. (b) Sketch of the trajectory at a time increment shorter than the Lagrangian integral timescale, where the length scales $l_{\|}$and $l_{\perp}$ follow form fitting a right triangle through the three subsequent points. (Reproduction of Fig. 1 in [17].)

approximated as

$$
\frac{l_{\perp}}{l_{\|}} \approx\left|\tan \left(\frac{\theta}{2}\right)\right| \approx\left|\frac{\theta}{2}\right|,
$$

using a Taylor expansion in the limit of small $\theta$. This approximation is expected to be valid for $\tau \ll T_{L}$ [17]. However, even for small values of $\tau$, larger angles of directional change can occur, for example, inside vortex tubes where a tracer can change its direction in a time interval that is of the order of the Kolmogorov timescale [13,31,32]. Since the scaling is derived for the average angle of directional change we expect that the influence of these kinds of rare events is minor. In the equation above $l_{\perp}$ corresponds to the distance traveled perpendicular to the initial displacement over a time interval $2 \tau$ and $l_{\|}$corresponds to the distance traveled parallel to the initial displacement in this time interval, as sketched in Fig. 1(b). These distances can be estimated as $l_{\perp} \approx 2 \tau^{2} a_{\perp}(t, \tau)$ and $l_{\|} \approx 2 \tau U(t, \tau)$, respectively, where $U(t, \tau)$ is the absolute value of the velocity and $a_{\perp}(t, \tau)$ is the absolute value of the acceleration component perpendicular to the velocity, both coarse grained over a time interval $\tau$. Without loss of generality, velocity and acceleration can be written in terms of positive random variables $\xi_{u}(t, \tau)$ and $\xi_{a}(t, \tau)$, with unit mean and variance, such that

$$
U(t, \tau)=\sigma_{u}(\tau) \xi_{u}(t, \tau), \quad a_{\perp}=\sigma_{a}(\tau) \xi_{a}(t, \tau),
$$

where $\sigma_{u}^{2}(\tau)$ and $\sigma_{a}^{2}(\tau)$ are the variances of $U(t, \tau)$ and $a_{\perp}(t, \tau)$, respectively. Substituting Eq. (5) into Eq. (4) and assuming that velocity and acceleration are uncorrelated (a reasonable assumption in HIT $[15,17]$ ) results in

$$
|\theta(t, \tau)| \approx 2 \tau \frac{\sigma_{a}(\tau)}{\sigma_{u}(\tau)}
$$

Now, two regimes are considered; in the first regime $\tau$ is much smaller than the Kolmogorov timescale $\tau \ll \tau_{\eta}$, and in the second regime $\tau$ is larger than the Kolmogorov timescale, but still much smaller than the Lagrangian integral timescale $\tau_{\eta}<$ $\tau \ll T_{L}$.
When $\tau \ll \tau_{\eta}$ we can assume that velocity and acceleration are constant over the time interval $\tau$, such that

$$
\Theta(\tau) \approx 2 \tau \frac{\sigma_{a}}{\sigma_{u}}
$$

In other words, the average angle $\Theta(\tau)$ is expected to scale as $\tau^{1}$ when $\tau \ll \tau_{\eta}$. In the inertial range, where $\tau_{\eta}<\tau \ll T_{L}$, Eq. (6) still holds, however, now $\sigma_{a}(\tau)$ depends on the time increment $\tau$. Using Kolmogorov phenomenology in the inertial range, dimensional analysis shows that $\sigma_{a}(\tau) \sim(\epsilon / \tau)^{1 / 2}$, with $\epsilon$ the energy dissipation rate $[17,33]$. By combining this expression for $\sigma_{a}(\tau)$ with Eq. (6), the average angle now becomes

$$
\Theta(\tau) \sim \tau^{1 / 2} \frac{\epsilon^{1 / 2}}{\sigma_{u}} \sim\left(\frac{\tau}{T_{L}}\right)^{1 / 2},
$$

using that $T_{L} \sim \sigma_{u}^{2} / \epsilon$. So, in the inertial range the average angle $\Theta(\tau)$ is expected to scale as $\tau^{1 / 2}$ in HIT. Note that in [17] this scaling is recovered for timescales up to almost 10 times the Kolmogorov timescale and for $\theta \lesssim 0.7$. The $\tau^{1 / 2}$ scaling, that is derived in the limit of small $\theta$ [Eq. (4)], is thus quite robust. For more details of the derivation above we refer the reader to [17].

For even larger timescales, where $\tau$ approaches the Lagrangian integral timescale, the convergence of the average angle $\Theta(\tau)$ depends on the domain configuration: the average angle between two randomly placed vectors in a 3D unbounded domain goes to $\pi / 2$ due to symmetry, while in a bounded domain this average angle approaches $2 \pi / 3$ due to confinement as explained in detail in [19].

The flow considered here, rotating $\mathrm{RBC}$, is anisotropic and inhomogeneous and therefore the assumptions of Gaussian velocity and acceleration statistics and uncorrelated velocity and acceleration are not obvious anymore. At small timescales the predictions above are still expected to hold, however, at large scales it is expected that inhomogeneity and anisotropy play a role and it is not a priori clear how this will influence the angular statistics.

\section{NUMERICAL AND EXPERIMENTAL METHODS}

\section{A. Direct numerical simulations}

The governing equations in the direct numerical simulations (DNS) are the incompressible Navier-Stokes and energy equations (including rotation) in the Boussinesq approximation:

$$
\begin{aligned}
\nabla \cdot \mathbf{u} & =0 \\
\frac{\partial \mathbf{u}}{\partial t}+(\mathbf{u} \cdot \nabla) \mathbf{u}+\frac{1}{\operatorname{Ro}} \hat{\mathbf{z}} \times \mathbf{u} & =-\nabla p+\sqrt{\frac{\operatorname{Pr}}{\operatorname{Ra}}} \nabla^{2} \mathbf{u}+T \hat{\mathbf{z}} \\
\frac{\partial T}{\partial t}+(\mathbf{u} \cdot \nabla) T & =\frac{1}{\sqrt{\operatorname{Pr} \mathrm{Ra}}} \nabla^{2} T
\end{aligned}
$$

with $\mathbf{u}$ the velocity vector, $t$ time, $p$ pressure, $T$ temperature, and $\hat{\mathbf{z}}$ the vertical unit vector. The equations are nondimensionalized using the height of the cell $H$ for length, the temperature difference $\Delta T$ for temperature, and $t_{c}=H / U$ for time, based on the free-fall velocity $U=\sqrt{g \alpha \Delta T H}$, where $g$ is the gravitational acceleration and $\alpha$ is the thermal 
expansion coefficient of the fluid. Now, the control parameters of this rotating $\mathrm{RBC}$ setup are the Rayleigh number $\mathrm{Ra}=$ $g \alpha \Delta T H^{3} /(\nu \kappa)$, the Prandtl number $\operatorname{Pr}=v / \kappa$, and the Rossby number Ro $=U /(2 \Omega H)$, with $v$ and $\kappa$ the kinematic viscosity and thermal diffusivity of the fluid, respectively, and $\Omega$ the rotation rate in $\operatorname{rad~s}^{-1}$. The equations above are solved in cylindrical coordinates with horizontal walls and a cylindrical sidewall. At all walls no-slip boundary conditions are applied, the temperature is fixed at the horizontal walls, and the sidewalls are adiabatic. The resolution is $512 \times 384 \times 512$ grid points in the azimuthal, radial, and axial directions, respectively. To deal with the singularity at $r=0$ (with $r$ the radius) the equations are solved in terms of $\left(u_{\phi}, r u_{r}, u_{z}\right)$ on a staggered grid, with $u_{\phi}, u_{r}$, and $u_{z}$ the velocity components in the radial, azimuthal, and axial directions, respectively. To ensure at least 10 grid points in the BLs, grid refinement is used in the axial and radial directions. For the discretization of the equations, a second-order finite difference scheme is used and for the time integration a third-order Runge-Kutta method is applied. The above numerical procedure is described in detail in $[34,35]$. In the RBC flow, $10^{6}$ fluid tracers are evolved, following the motion of the fluid with high accuracy. A trilinear interpolation scheme is used to interpolate the fluid velocity from the grid points to the position of the particle. For the time integration of the particle motion, a second-order Adams-Bashforth scheme is used. The Rayleigh and Prandtl numbers are fixed to $\mathrm{Ra}=1.3 \times 10^{9}$ and $\mathrm{Pr}=6.7$, while the Rossby number range is $0.05 \leqslant$ Ro $\leqslant \infty$.

Lagrangian statistics of these tracer trajectories are collected both in the center and near the top plate of the cell in different measurement volumes. In the center, a measurement volume of size $0.5 \mathrm{H} \times 0.5 \mathrm{H} \times 0.5 \mathrm{H}$ is considered as sketched in Fig. 2 (gray cube). Inside this measurement volume we have approximately $1.6 \times 10^{5}$ tracers, based on a uniform distribution of tracers within the RBC cell. To give an idea of the convergence of the statistics with the size of the measurement volume, the average angle of directional change computed in a volume of $0.2 \mathrm{H} \times 0.2 \mathrm{H} \times 0.2 \mathrm{H}$ deviates about $30 \%$ from the values corresponding to the measurement volume of $0.5 \mathrm{H} \times 0.5 \mathrm{H} \times 0.5 \mathrm{H}$ and for a volume of $0.4 \mathrm{H} \times$ $0.4 H \times 0.4 H$ this deviation already reduced to about $7.5 \%$, when focusing on the same time range. Furthermore, in the measurement volume of $0.5 \mathrm{H} \times 0.5 \mathrm{H} \times 0.5 \mathrm{H}$, trajectories are tracked long enough to investigate the convergence of the average angle of directional change at large timescales. A second measurement volume is placed close to the top plate. In the DNS, the boundary layer is accessible, such that we can explicitly measure the effect of BL dynamics on the geometry of the tracer trajectories at different timescales. Therefore, the numerical measurement volume near the top plate is subdivided into a nonboundary layer region (non-BL, dark green rectangular parallelepiped in Fig. 2) and the viscous boundary layer region $(\mathrm{BL}$, light green rectangular parallelepiped in Fig. 2), where the sizes of these volumes are $0.5 \mathrm{H} \times 0.5 \mathrm{H} \times$ $\left(0.25-\delta_{u}\right) H$ and $0.5 H \times 0.5 H \times \delta_{u} H$, respectively, with $\delta_{u}$ the viscous BL thickness. $\delta_{u}$ is determined as the position of the maximum horizontal root-mean-square velocity and varies from $\delta_{u}=0.0299 H$ for Ro $=\infty$ to $\delta_{u}=0.0058 H$ for Ro $=0.05$. Given that tracers are distributed uniformly, the number of tracers inside the non-BL and BL measurement

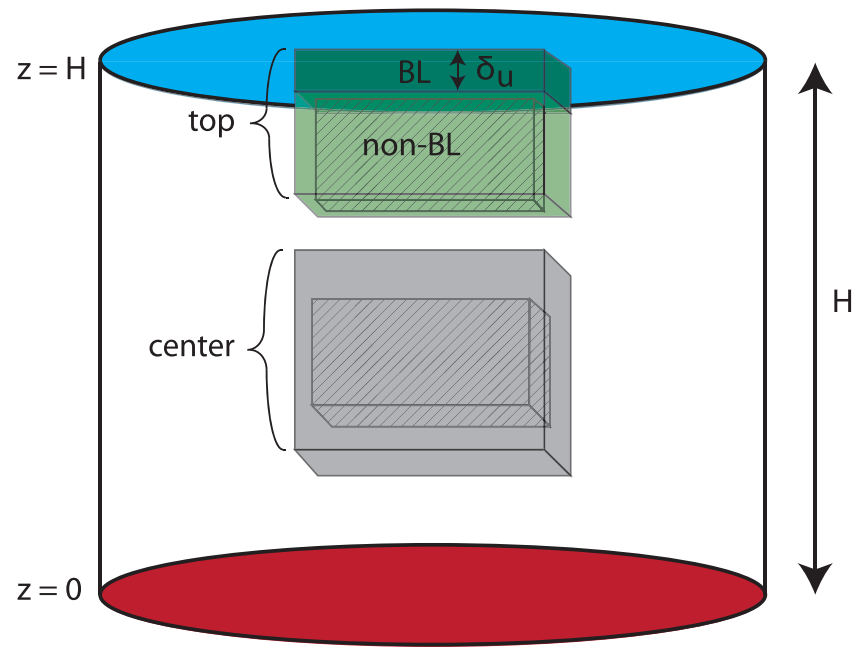

FIG. 2. Sketch of the measurement volumes. The gray cube in the center represents the measurement volume in the DNS of size $0.5 H \times 0.5 H \times 0.5 H$, in the $x, y$, and $z$ directions, respectively, while the hatched part shows the measurement volume used in the experiments of size $0.4 H \times 0.3 H \times 0.25 H$, with $H=200 \mathrm{~mm}$ being the cell height. The green hatched rectangular parallelepiped at the top represents the experimental measurement volume of size $0.4 H \times 0.3 H \times 0.25 H$. In the DNS this volume is subdivided in the vertical direction into a nonboundary layer (non-BL) part of size $0.5 H \times 0.5 H \times\left(0.25 H-\delta_{u} H\right)$ (dark green upper part), and a boundary layer (BL) part of size $0.5 H \times 0.5 H \times \delta_{u} H$ (light green lower part), with $\delta_{u}$ the dimensionless viscous boundary layer thickness, which varies between $\delta_{u}=0.0299 H$ and $0.0058 H$, depending on the rotation rate [11]. Note that this sketch is not in real scale, particularly the BL size is enlarged for visibility reasons.

volumes is $7-8 \times 10^{4}$ and $1-9 \times 10^{3}$, respectively, depending on $\delta_{u}$. Trajectories are reconstructed such that they start and end in the measurement volume: this means that each time a tracer enters the measurement volume it starts a new trajectory and a tracer leaving the measurement volume terminates a trajectory.

The Kolmogorov timescale $\tau_{\eta}$ and the Lagrangian integral timescale $T_{L}$ of the trajectories are different in the center compared to the volume close to the top plate and additionally depend on the rotation rate. Their values are reported in Table I, where $\tau_{\eta}=(\nu / \epsilon)^{1 / 2}$, based on the average energy dissipation $\epsilon$ in the different measurement volumes and the Lagrangian integral timescale is estimated as the time at which the second-order Lagrangian velocity structure function $S_{i, 2}=\left\langle\left[u_{i}(t+\tau)-u_{i}(t)\right]^{2}\right\rangle$, with $i$ the horizontal or vertical component of the velocity, has converged to its plateau value. To compute the structure functions in the horizontal direction, the components in the $x$ and $y$ directions are averaged, such that $S_{x y, 2}=\left(S_{x, 2}+S_{y, 2}\right) / 2$. The error in the Lagrangian inertial timescales reported in Table I is maximum $20 \%$, based on different thresholds used to determine the convergence. Except for $\mathrm{Ro}=0.05$ and $\mathrm{Ro}=10$, the Kolmogorov timescales are computed from previous DNS data $[11,24]$ with a slightly different grid resolution of $512 \times 257 \times 512$ grid points. The values are, however, expected to be the same for the resolution used here. 
TABLE I. Kolmogorov timescales $\tau_{\eta}$ and Lagrangian integral timescales $T_{L}$ of tracer particles in rotating Rayleigh-Bénard convection. Timescales are reported at different Rossby numbers and for both the center measurement volume and the measurement volume close to the top plate, excluding the BL. The Lagrangian integral timescale is reported for both the horizontal and the vertical directions, given by $T_{x y}$ and $T_{z}$, respectively. All timescales are nondimensionalized by $t_{c}=H / U$.

\begin{tabular}{cccccccccccc}
\hline \hline \multicolumn{1}{c}{ Ro } & 0.05 & 0.1 & 0.2 & 0.5 & 1 & 2.5 & 3 & 5 & 10 & $\infty$ \\
\hline Center & $\tau_{\eta}$ & 0.41 & 0.36 & 0.35 & 0.36 & 0.36 & 0.39 & 0.43 & 0.51 & 0.46 & 0.52 \\
& $T_{x y}$ & 14.3 & 6.2 & 23.1 & 15.3 & 4.7 & 7.1 & 5.7 & 4.9 & 7.4 & 3.5 \\
& $T_{z}$ & 18.4 & 7.7 & 10.9 & 9.1 & 8.6 & 8.1 & 7.0 & 5.5 & 6.1 & 4.9 \\
Top & $\tau_{\eta}$ & 0.33 & 0.32 & 0.32 & 0.33 & 0.33 & 0.35 & 0.37 & 0.39 & 0.40 & 0.40 \\
& $T_{x y}$ & 18 & 3.2 & 4.0 & 9.3 & 5.2 & 6.2 & 8.4 & 4.2 & 7.9 & 13.7 \\
& $T_{z}$ & 10.9 & 22.0 & 13.4 & 8.0 & 4.5 & 4.0 & 4.7 & 8.6 & 2.7 & 2.7 \\
\hline \hline
\end{tabular}

\section{B. Experiments}

The DNS results are compared to experiments of rotating $\mathrm{RBC}$, which are described in detail in $[11,20]$. In these experiments a Plexiglas cylinder, filled with water and with height $H=200 \mathrm{~mm}$ and diameter $D=200 \mathrm{~mm}$, is heated from below and cooled from above. The bottom plate is made of copper and includes an electrical resistance heater, while the cooling chamber at the top consists of a transparent sapphire plate, assuring optical visibility and large thermal conductivity (and thus a homogeneous temperature distribution in the sapphire plate). Between the plates a temperature difference of $\Delta T=10 \mathrm{~K}$ is applied. Neutrally buoyant particles, with a density of $\rho_{p}=1002 \mathrm{~kg} \mathrm{~m}^{-3}$ and a diameter of $d_{p}=75-90 \mu \mathrm{m}$ (smaller than the Kolmogorov length that is of the order of millimeters), are inserted in the carrier fluid that has a mean density of $\rho_{f}=998 \mathrm{~kg} \mathrm{~m}^{-3}$. The Stokes number of the particles is $\mathrm{St}=\tau_{p} / \tau_{\eta} \approx 5 \times 10^{-4}$, with $\tau_{p}=d_{p}^{2} \rho_{p} /\left(18 v \rho_{f}\right)$ the particle response time, assuring that they behave as tracer particles. Particles are tracked by four CCD cameras, with a resolution of $1600 \times 1200$ pixels and a frequency of 30 $\mathrm{Hz}$, placed above the RBC cell. From the recorded images, trajectories are reconstructed using the 3D-Particle Tracking Velocimetry algorithm developed at ETH Zürich [36,37]. The complete setup, including the optical tracking system, is placed on a rotating table in order to vary the rotation rate $\Omega$. Like in the DNS, the Rayleigh and Prandtl numbers are $\mathrm{Ra}=$ $1.3 \times 10^{9}$ and $\operatorname{Pr}=6.7$ (corresponding to water), respectively. The rotation rate is varied between $0(\operatorname{Ro}=\infty)$ and 1.65 $\operatorname{rad~s}^{-1}($ Ro $=0.1)$.

Two different measurement volumes of size $80 \times 60 \times 50$ $\mathrm{mm}^{3}$ are used in the experiments: one in the center of the cell and one near the top plate, as shown in the sketch in Fig. 2 as the hatched rectangular parallelepipeds. These volumes are smaller than those used in the DNS, resulting in smaller statistical samples and shorter trajectories in the experimental data compared to the DNS data. Due to the calibration method, there is an offset between the plate and the top measurement volume of about $1 \mathrm{~mm}$, where 3D positioning of the tracer particles is not possible. Compared to the viscous boundary layer (BL) thickness $\delta_{u}$ the offset varies from $0.167 \delta_{u}$ for Ro $=\infty$ to $0.617 \delta_{u}$ for Ro $=0.1$ [11]. This offset, together with the fact that particles have a higher density than the water on average [and thus a positive settling velocity $w=$ $\left.\left(\rho_{p}-\rho_{f}\right) g d_{p}^{2} /\left(18 v \rho_{f}\right) \approx 0.018 \mathrm{~mm} \mathrm{~s}^{-1}\right]$, results in a scarcity of data points inside the boundary layer. Therefore, we treat experimental data collected in the measurement volume near the top plate as if the BL is excluded.

Since experiments and DNS are performed with the same parameters, the Kolmogorov timescales are similar. Also, the Lagrangian integral timescales are expected to be similar given that the Stokes number of the particles in the experiments is small, i.e., $\mathrm{St} \approx 5 \times 10^{-4}$.

\section{RESULTS}

\section{A. NonRotating Rayleigh-Bénard convection}

First, we will discuss the angle of directional change of tracer trajectories in nonrotating RBC. The Kolmogorov times and Lagrangian integral timescales of nonrotating RBC are as reported in Table I.

\section{Probability density functions of the angle of directional change}

Probability density functions (PDFs) of the angle of directional change, measured in the center, are compared to PDFs measured near the top plate. We start from the central measurement volume, where the flow is closest to HIT [20,22,38]. In Fig. 3(a), a good agreement between DNS and experiments is found for the PDFs of $\theta$, indicated by $P_{\theta}$. The PDFs show a peak around $\theta=0$ for small $\tau$ and approach the distribution $P_{\theta, \tau \rightarrow \infty}=\frac{1}{2} \sin \theta$, corresponding to the angle between two randomly chosen vectors in 3D space, for large $\tau$. This trend was also observed in [17] for HIT.

In the DNS, we also consider PDFs of $Q=1-\cos \theta$, which we will indicate by $P_{Q}$. This quantity $Q$ gives a direct measure for the directional change, being zero for very small angles (particles going straight) and two for a complete reversion of the direction (corresponding to $\theta=\pi$ ). Given that $P_{\theta}$ goes to $P_{\theta, \tau \rightarrow \infty}(\theta)=\frac{1}{2} \sin \theta$ for large time increments, $P_{Q}$ is expected to be equidistributed in that limit. In Fig. 3(c), $P_{Q}$ is indeed converging to the uniform distribution $P_{Q, \tau \rightarrow \infty}(Q)=\frac{1}{2}$ for larger $\tau$. As shown in [17], an analytical prediction for the shape of $P_{Q}$ in the limit of $\theta \rightarrow 0$ can be derived. First, a Taylor expansion of $1-\cos \theta$ gives

$$
1-\cos \theta(t, \tau) \approx \frac{1}{2} \theta^{2}(t, \tau) \approx 2 \tau^{2} \frac{\sigma_{a}^{2}(\tau) \xi_{a}^{2}}{\sigma_{u}^{2}(\tau) \xi_{u}^{2}},
$$

where Eqs. (5) and (6) are used. Then, by assuming $u$ and $a$ to be uncorrelated and to follow Gaussian statistics, $\xi_{u}^{2}$ and $\xi_{a}^{2}$ are expected to follow a $\chi$-squared distribution with three and two components, respectively. As derived in [17] this means that $P_{Q}$ can be described by a Fisher distribution $F_{n, m}$, with $n=3$ and $m=2$. For HIT, PDFs for different values of $\tau$ are shown to collapse when normalized by $\gamma_{2,3}(\tau)=\Theta^{2}(\tau) / 3$ (following from the exact definition of $F_{2,3}$ using the $\chi$-squared distributions), where $\Theta(\tau)=\langle|\theta(t, \tau)|\rangle$. In Fig. 3(e) we show the normalized PDFs of $Q$ and find the curves to collapse onto the analytical prediction of the Fisher distribution $F_{2,3}$. The agreement is particularly good for small values of $\tau$, but even for larger values of $\tau$, where assumptions of Gaussian statistics and small $\theta$ are violated, the agreement is still acceptable. 


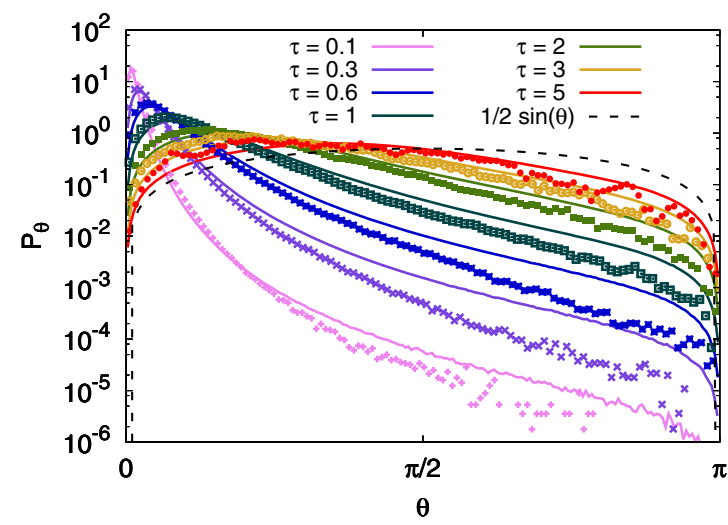

(a) center

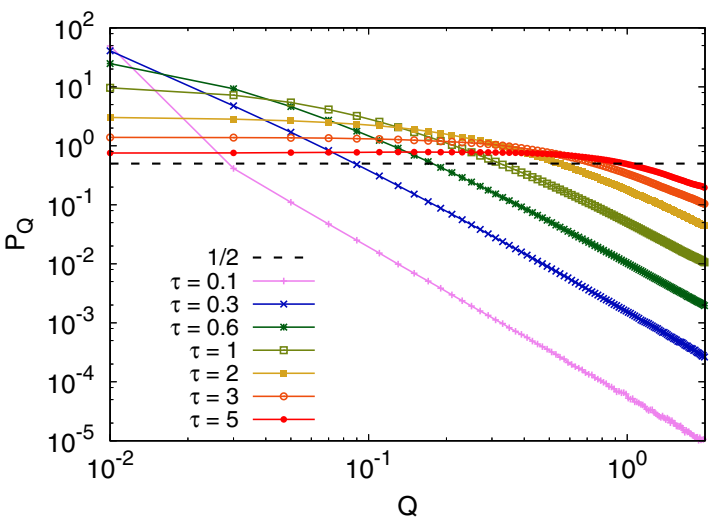

(c) center

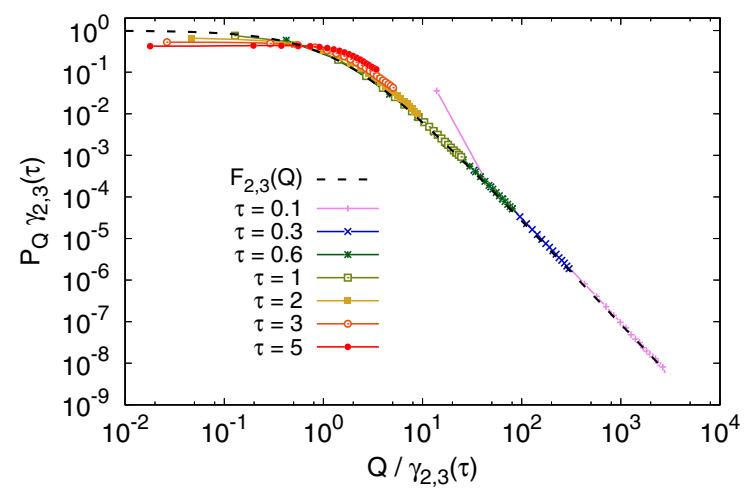

(e) center

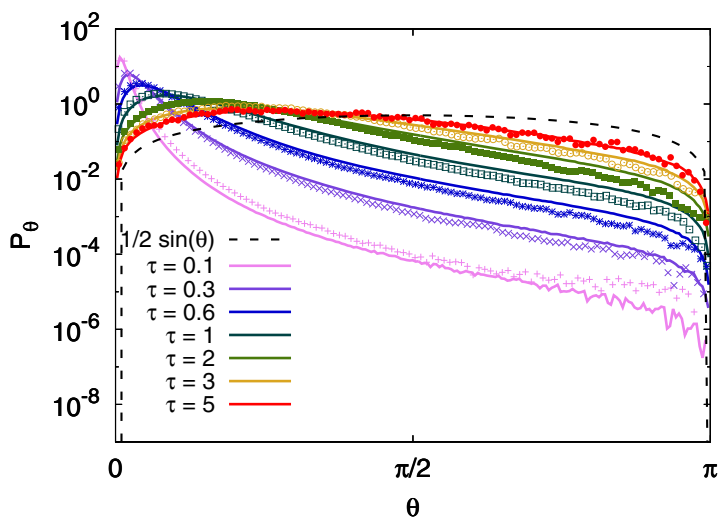

(b) top, non-BL

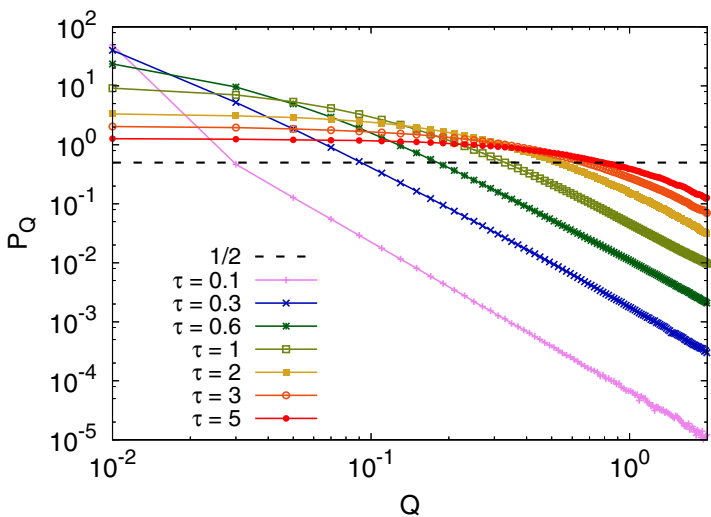

(d) top, non-BL

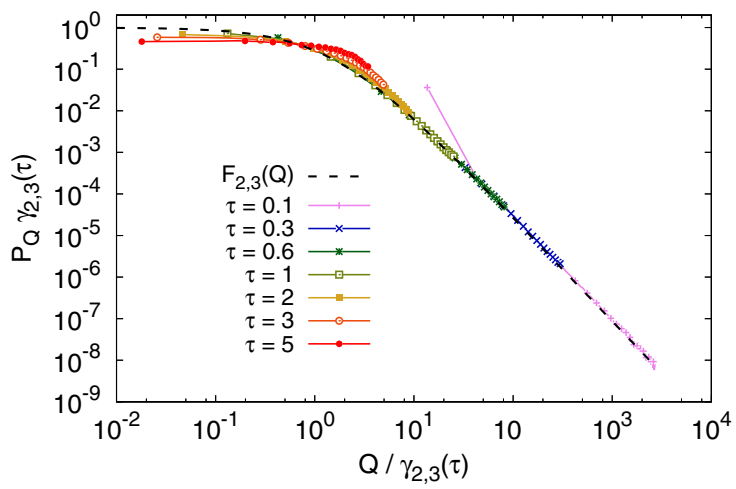

(f) top, non-BL

FIG. 3. Top: PDFs of the angle $\theta, P_{\theta}$, for different (nondimensional) time increments $\tau$ measured in (a) the center and (b) the nonboundary layer (non-BL) region near the top plate in both experiments (symbols) and direct numerical simulations (DNS, lines) of nonrotating RayleighBénard convection. Focusing on the right part of the figures, $\tau$ is increasing from bottom to top. Center: PDFs of $Q=1-\cos \theta, P_{Q}$, for the DNS data now represented by lines with symbols in (c) the center and (d) the non-BL region. Bottom: $P_{Q}$, but now normalized by $\gamma_{2,3}(\tau)=2(\tau) / 3$, with the average angle of directional change, for (e) the center and (f) the non-BL region. The black dashed line shows a Fisher distribution $F_{2,3}$. For clarity, symbols are plotted in each 5 data points.

Near the top plate, inhomogeneity and anisotropy are expected to play a role. To investigate how this affects the angular statistics we show $P_{\theta}$, measured near the top plate in Fig. 3(b), where in the DNS the boundary layer is excluded. We again find a good agreement between DNS and experiments, given that in the experiments very little measurement points are found inside the viscous BL as mentioned before in Sec. III B. Distributions of $P_{\theta}$ [Fig. 3(b)] and $P_{Q}$ [Fig. 3(d)] are similar as the corre- sponding distributions measured in the center and again $P_{Q}$ recovers the Fisher distribution $F_{2,3}$, when properly normalized. So, statistics of the angle $\theta$ recovers the behavior found for HIT, even in the anisotropic and inhomogeneous flow studied here. This was already shown for the limit of $\tau \rightarrow 0$ in [16], where curvature and torsion PDFs in RBC where shown to recover the HIT predictions, as long as the BL was excluded from the measurement volume. Our results suggest that for time- 
dependent quantities like $\theta$ this assumption can be extended to larger timescales, even up to the Lagrangian integral timescale.

As in [16] we expect different behavior inside the viscous BLs, where a strong mean flow is present in the regime with presence of the LSC. In Figs. 4(a) and 4(b) we show that both $P_{\theta}$ [Fig. 4(a)] and $P_{Q}$ [Fig. 4(b)] measured in the BL using DNS, do not approach the uniform distributions (dashed black lines) anymore like in the bulk. Instead, in Fig. 4(a), a flat wide maximum develops around $\theta=0$ for larger $\tau$, consistent with the expectation that trajectories are straighter inside the BL due to the horizontal mean flow. The presence of this strong mean horizontal flow can also be seen as a reduction of the dimensionality, such that the velocity and normal acceleration do not have three and two components, but rather one and two components, respectively. Indeed, when we now compute $P_{Q}$, normalized by $\gamma_{1,2}(\tau)=\Theta^{2}(\tau) / 4$, a collapse on a Fisher distribution $F_{1,2}$ is observed in Fig. 4(c), supporting the proposition that the effective number of components of velocity and acceleration has decreased. Note that the definition of $F_{1,2}$ results in a different normalization factor for $P_{Q}\left[\gamma_{1,2}(\tau)=\Theta^{2}(\tau) / 4\right]$ than the definition of $F_{2,3}$ [where $\gamma_{2,3}(\tau)=\Theta(\tau)^{2} / 3$ ]. In Fig. 4(c), the steep peaks at the righthand-side end of the curves correspond to larger values of $\theta$, where the Taylor expansion of Eq. (12) does not hold anymore.

\section{Average angle of directional change}

The multiscale temporal behavior of the flow can be investigated by measuring the average angle $\Theta$ as a function of $\tau$. As explained in Sec. II, $\Theta$ is expected to scale as $\Theta \sim \tau$ for $\tau \ll \tau_{\eta}$ and as $\Theta \sim \tau^{1 / 2}$ for $\tau_{\eta}<\tau \ll T_{L}$ in HIT. In Fig. 5(a), a good agreement between experiments and DNS is found for $\Theta(\tau)$, provided that the BL is excluded in the DNS. The average angle $\Theta$ converges to $2 \pi / 3$ for large $\tau$, consistent with the prediction for confined domains in [19]. The confinement comes from the sampling method, based on sampling trajectories such that they are inside the measurement volume over their full life span. Even though this measurement volume is not bounded by physical walls, the angles at subsequent points along the trajectories are not equidistributed when the corresponding separation lengths between the points become of the order of the measurement volume. In [19], the convergence to $2 \pi / 3$ is reproduced by placing three random points in a confined domain, using Monte Carlo simulations, and computing the angle between the two line segments connecting these points. These simulations only capture the geometrical effects of confinement and disregard flow-dependent effects, like the modification of the flow at the walls. The fact that the convergence to $2 \pi / 3$ is recovered thus indicates that it is a purely geometrical effect, like in our data sampling.

When focusing on the scaling of $\Theta$ with $\tau$ in Fig. 5(a), we recover the ballistic scaling of $\Theta \sim \tau$ in the viscous regime where $\tau \ll \tau_{\eta}$, as long as the BL is excluded. For $\tau>\tau_{\eta}$, a $\tau^{1 / 2}$ scaling is predicted for HIT, linked to the Kolmogorov scaling of the energy spectrum in the inertial range. In the RBC setup studied here, the Taylor-based Reynolds number is relatively low: $\operatorname{Re}_{\lambda} \approx 39$, where $\operatorname{Re}_{\lambda}=u^{\prime 2} \sqrt{15 /(\epsilon \nu)}$, with $u^{\prime}=\left(u_{x}^{\mathrm{rms}}+u_{y}^{\mathrm{rms}}+u_{z}^{\mathrm{rms}}\right) / 3$. Consequently, one cannot expect a Kolmogorov scaling of the energy spectrum in the inertial

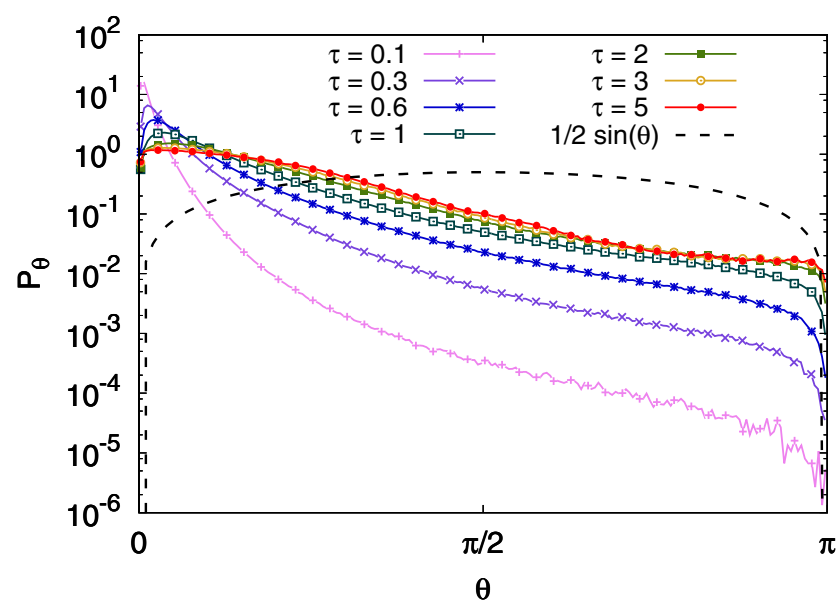

(a)

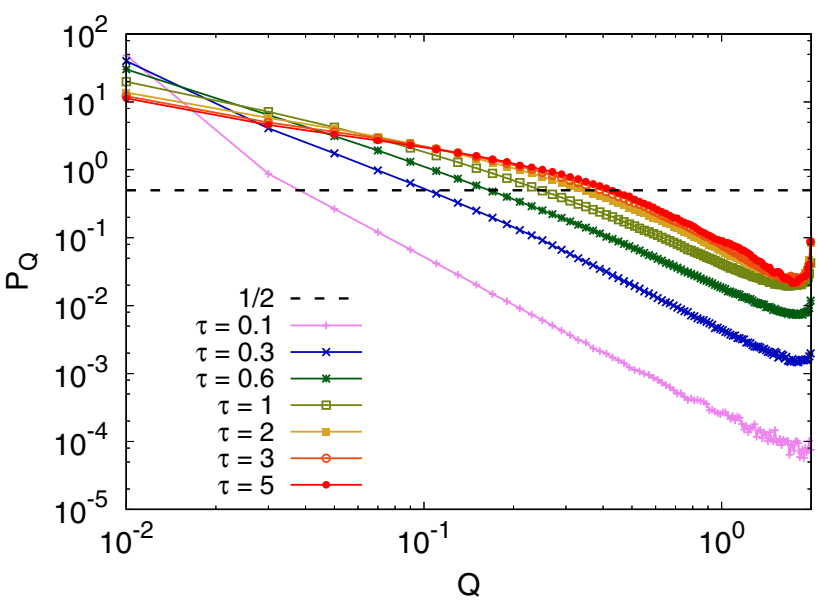

(b)

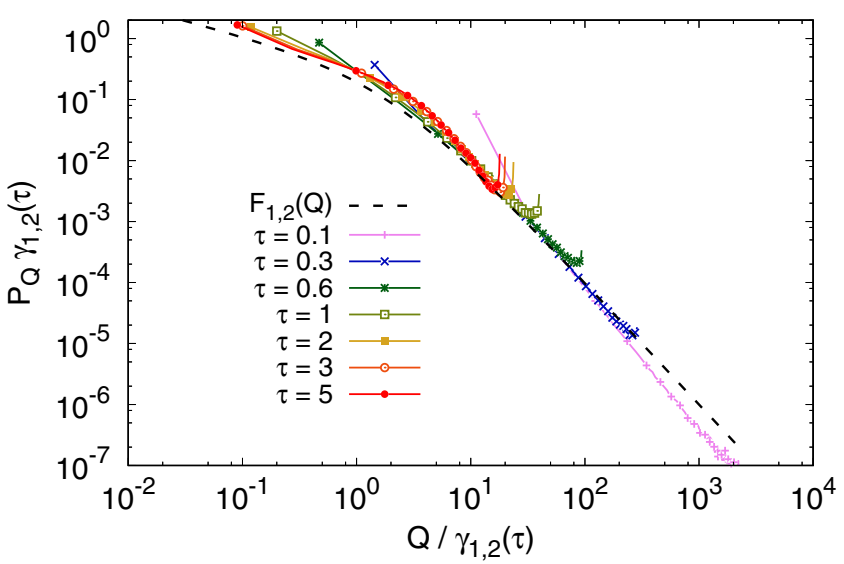

(c)

FIG. 4. (a) PDFs of the angle $\theta, P_{\theta}$, for different (nondimensional) time increments $\tau$, measured in the viscous BL at the top plate for direct numerical simulations (DNS) of nonrotating Rayleigh-Bénard convection. (b) PDFs of $Q=1-\cos \theta, P_{Q}$, for DNS. (c) $P_{Q}$, but now normalized by $\gamma_{1,2}(\tau)=\Theta^{2}(\tau) / 4$, with $\Theta$ the average angle of directional change, together with a Fisher distribution $F_{1,2}$ (black dashed line). For clarity, symbols are added to the lines each 5 data points in panels (a) and (c) and each data point in panel (b). 


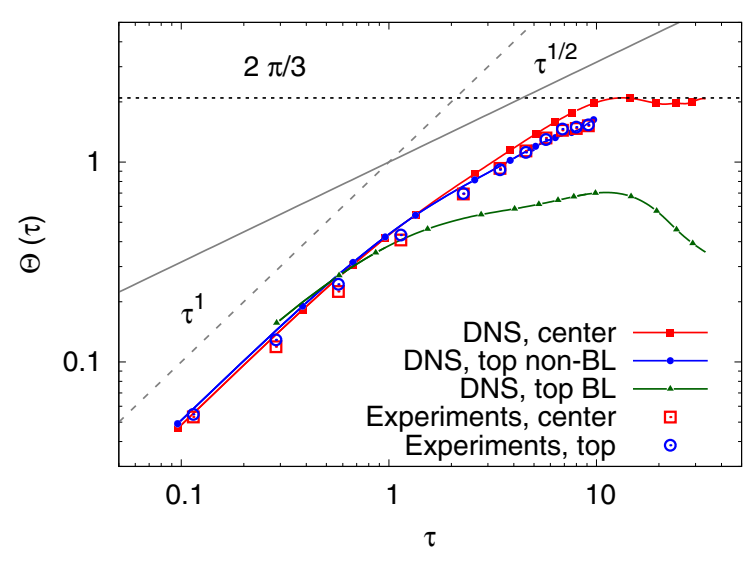

(a)

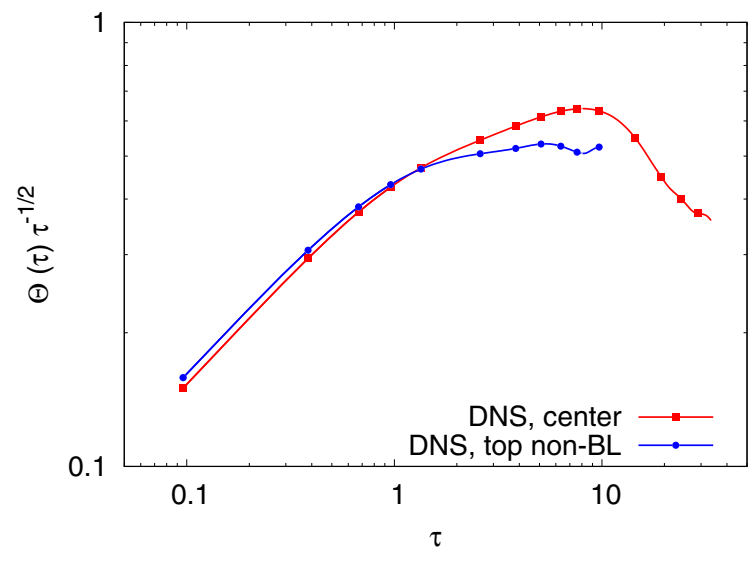

(b)

FIG. 5. (a) The average angle of directional change $\Theta(\tau)=\langle|\theta(t, \tau)|\rangle$ as a function of the nondimensional time increment $\tau$, for both DNS (lines with closed symbols) and experiments (open symbols), measured in the center (red squares) and near the top plate (blue circles) of the Rayleigh-Bénard cell. In the DNS the top measurement volume is subdivided in a nonboundary layer (non-BL) and boundary layer (BL) region, indicated by the green lines with triangles. (b) $\Theta(\tau)$, normalized by $\tau^{1 / 2}$, measured using DNS in the center (red lines with squares) and the non-BL region (blue lines with circles). For the DNS symbols are added to the lines for visibility with an interval depending on $\tau$.

range, possibly explaining why the $\tau^{1 / 2}$ is not recovered very well. We do, however, observe a transition in the scaling from $\tau^{1}$ for $\tau \ll \tau_{\eta}$ to a lower scaling exponent in the regime of $\tau_{\eta}<\tau<T_{L}$. This is also clearly visible when focusing on $\Theta(\tau)$, compensated by $\tau^{1 / 2}$ in Fig. 5(b). Although the presence of a plateau is lacking, especially in the center, there is clearly a transition from one scaling regime to another at $\tau \approx 1$.

While the $\Theta(\tau)$ curves for the center and the non-BL volume mostly overlap, the average angle in the Prandtl-Blasius BL behaves completely different. For larger $\tau$, the values of $\Theta$ are lower as a result of the strong horizontal mean flow present in the Prandtl-Blasius BL [11,39]. Since the measurement volume used in the BL is rather small, it is important to distinguish between the effect of the mean flow and the effect of the confinement. Therefore, we measured $\Theta(\tau)$ in a measurement volume of the size of the BL measurement volume $\left(0.5 H \times 0.5 H \times \delta_{u} H\right)$, but positioned in the center. Without showing the figures here, we verified that the strong decrease of $\Theta(\tau)$ for larger $\tau$ was only found in the BL and not in the center (when using this BL measurement volume), confirming that it is a flow-dependent feature.

\section{B. Rotating Rayleigh-Bénard convection}

Now, we include rotation and investigate how it affects the geometry of the flow by measuring the angle of directional change of tracer trajectories at different timescales and at different Rossby numbers. Note that the Kolmogorov timescale and the Lagrangian timescale depend on the rotation rate as reported in Table I.

\section{Probability density functions of the angle of directional change}

First, we investigate the effect of rotation on the directional change $Q$ by analyzing $P_{Q}$ at different Rossby numbers and different values of $\tau$ in Fig. 6, for both the center and the top, non-BL, measurement volumes. The normalized curves all collapse on the Fisher distribution $F_{2,3}$, indicating that the analytical prediction of Sec. IV A is still valid. This means that the assumption of noncorrelated velocity and acceleration and the assumption of Gaussian statistics remain valid in the bulk of the rotating RBC flow. For instantaneous curvature and torsion statistics, this was already shown in [16], however, here we suggest that this also holds for larger timescales up to the Lagrangian integral timescale of multitimescale processes.

The BL is of the Ekman type for Ro $\lesssim 2.5$ and PrandtlBlasius type for Ro $\gtrsim 2.5$ [11]. Both types of BLs are characterized by different flow structures and we want to understand whether the $\mathrm{BL}$ transition is visible in the angular statistics of tracer trajectories. $P_{\theta}$, measured in the Prandtl-Blasius BL where a strong mean horizontal flow is present, reveals a higher probability of low values of $\theta$ in Fig. 4(a), while in the Ekman BL the probability of larger $\theta$ for larger $\tau$ has increased in Fig. 6(c). This is possibly a signature of the spiraling motions of fluid [40], characterizing the Ekman BL flow. The described trend is also directly visible in $P_{Q}$; there is an enhanced probability on larger values of $Q$ inside the BL in the rotating case, evident when comparing Figs. 4(b) and 6(d). In Figs. 6(c) and 6(d) we only show PDFs for one rotation rate, Ro $=0.1$, but we verified that similar statistics are retrieved for other rotation rates in the regime of Ro $\lesssim 2.5$. To understand the effective dimensionality of the $\mathrm{BL}$ flows, we normalize $P_{Q}$ and compare it to a Fisher distribution with either two and three components $F_{2,3}$ or one and two components $F_{1,2}$. For Ro $=\infty$, a reduction of the effective number of components due to the mean horizontal flow in the BL was already observed in Sec. IV A. The Ekman BL is not characterized by a strong horizontal mean flow, but by spiraling motion of fluid. To understand the effective dimensionality in the Ekman BL, we compare $P_{Q}$, normalized by $\Theta^{2}(\tau)$, to both $F_{2,3}$ and $F_{1,2}$ in Fig. 6(e), where $\tau$ is increased from right to left. ${ }^{1}$ For small

\footnotetext{
${ }^{1}$ For $F_{1,2}$ the normalization factor is $\gamma_{1,2}=\Theta^{2}(\tau) / 4$, while for $F_{2,3}$ the normalization factor is $\gamma_{2,3}=\Theta^{2}(\tau) / 3$. In order to account for these different normalization factors in Fig. 6(e), we compare $P_{Q}$, normalized by $\Theta(\tau)^{2}$, to $4 F_{1,2}(Q / 4)$ and $3 F_{2,3}(Q / 3)$, respectively.
} 


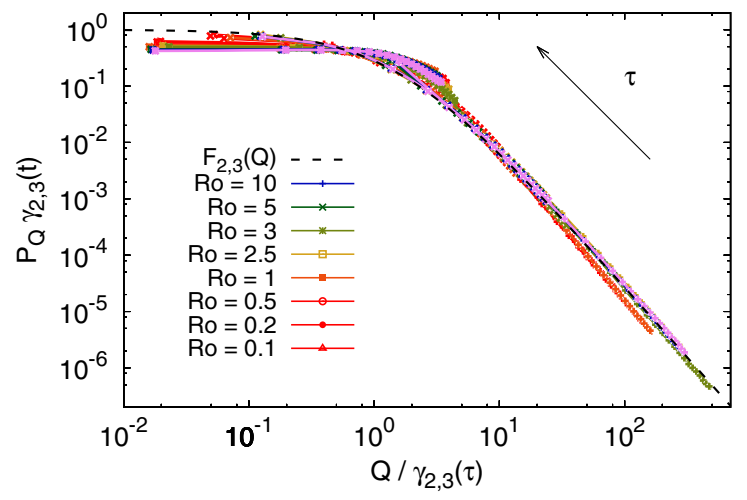

(a) center

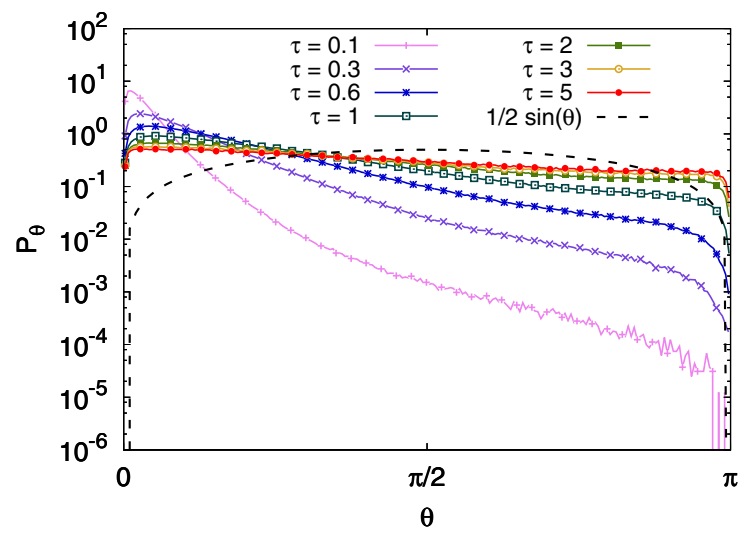

(c) top, BL, Ro $=0.1$

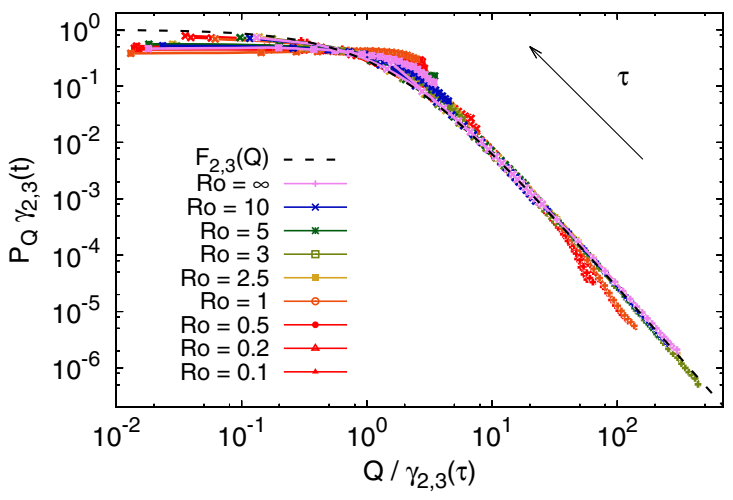

(b) top, non-BL

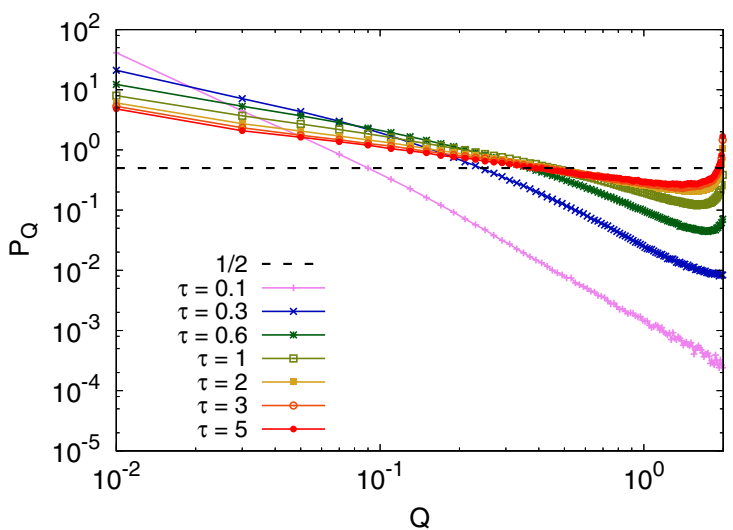

(d) top, BL, Ro $=0.1$

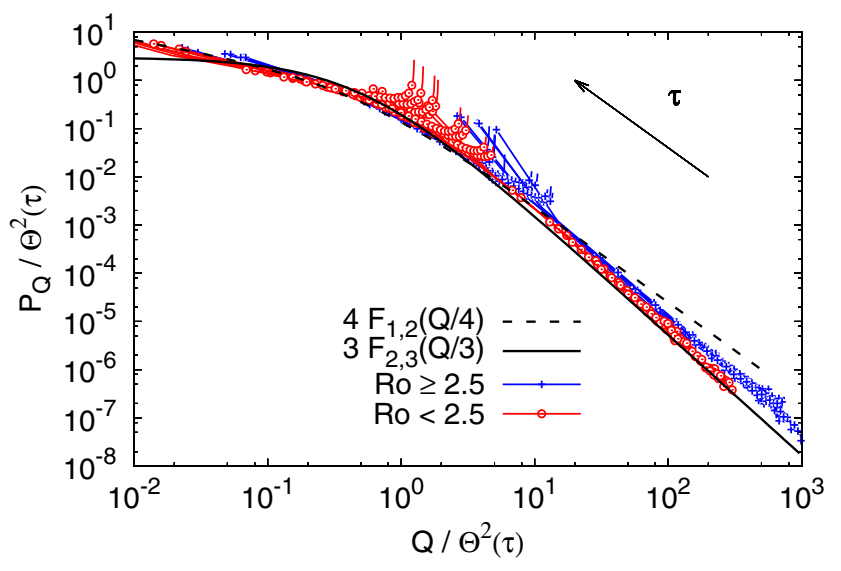

(e) top, BL

FIG. 6. Top: PDFs of $Q=1-\cos \theta, P_{Q}$, for different Rossby numbers Ro, measured using direct numerical simulations (DNS) in (a) the center and (b) the nonboundary layer (non-BL) region near the top plate of the Rayleigh-Bénard cell. PDFs are normalized by $\gamma_{2,3}(\tau)=\Theta^{2}(\tau) / 2$, with $\Theta(\tau)$ the average angle of directional change. The black dashed line represents the Fisher distribution $F_{2,3}$. For each Ro time increments $\tau=0.3,1$, and 5 are shown. Center: (c) $P_{\theta}$ and (d) $P_{Q}$, measured in the BL for Ro $=0.1$. Bottom: (e) $P_{Q}$, normalized by $\Theta^{2}(\tau)$, measured in the BL for Ro $\geqslant 2.5$ (blue lines with crosses) and Ro $<2.5$ (red lines with circles). For each Ro, $\tau=0.1,1$ and 5 are shown. The black solid and black dashed lines represent $3 F_{2,3}$ and $4 F_{1,2}$, respectively. Symbols are added to lines each data point in panel (d) and each 5 data points in all other panels for visibility.

$\tau$ we observe that $P_{Q}$ is closer to the Fisher distribution $F_{2,3}$ in the Ekman BL (red curves) while $P_{Q}$ in the Prandtl-Blasius BL (blue lines) starts to approach the Fisher distribution $F_{1,2}$. So, for small $\tau$, the reduction in the dimensionality is only found in the Prandtl-Blasius BL, characterized by the strong mean horizontal flow. For larger $\tau$, however, curves in both the Prandtl-Blasius and the Ekman BL collapse on the Fisher distribution $F_{1,2}$. This is probably the result of trajectories being reconstructed in a thin measurement volume with a small vertical dimension (of the size of the BL thickness), compared to the horizontal dimensions, reducing the components of the normal acceleration and velocity of trajectories at large 


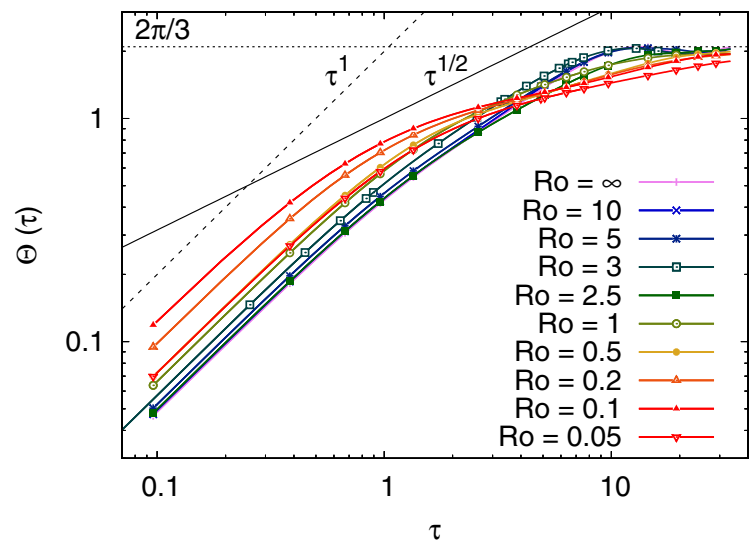

(a) center

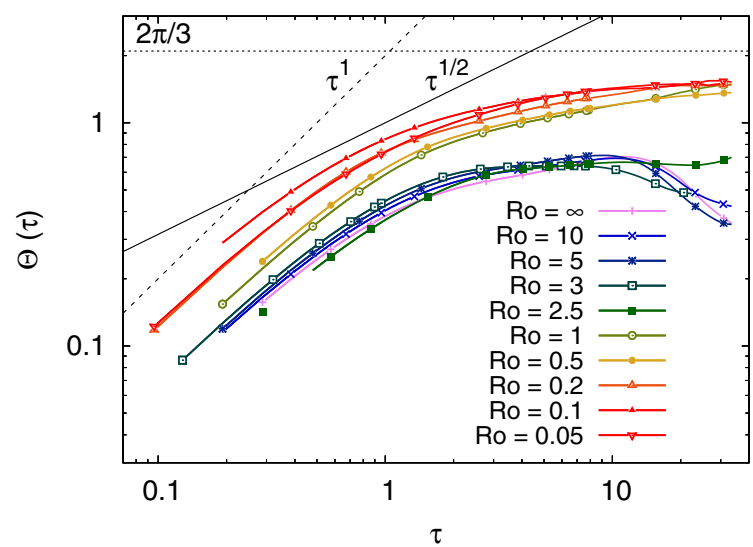

(c) top, BL

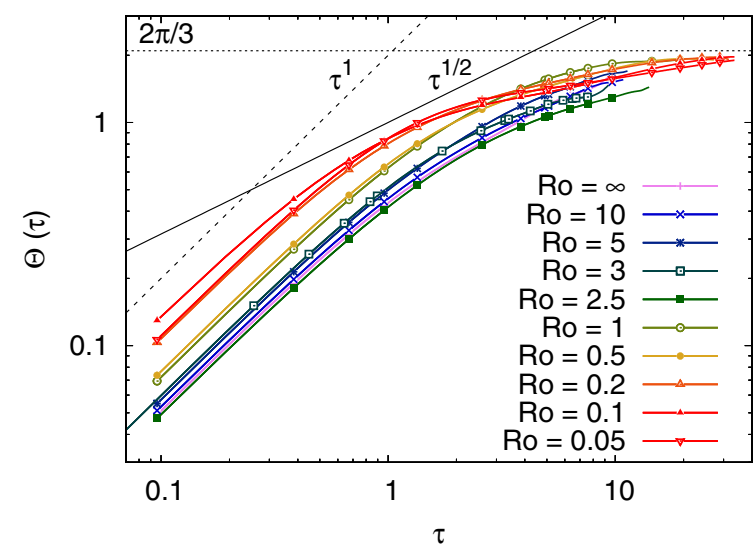

(b) top, non-BL

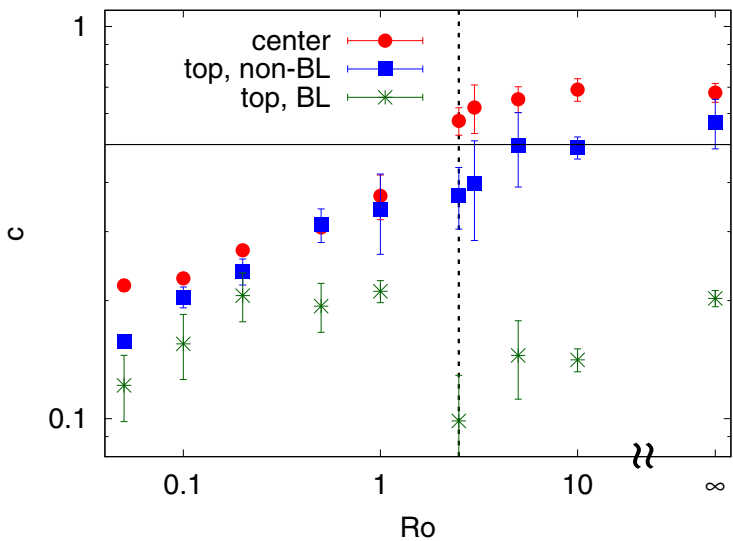

(d) scaling exponents

FIG. 7. The average angle $\Theta(\tau)=\langle|\theta(t, \tau)|\rangle$ as a function of $\tau$ (in dimensionless time units), measured using DNS in (a) the center, (b) the nonboundary layer (non-BL) region, and (c) the boundary layer (BL) region near the top plate of the Rayleigh-Bénard cell. Symbols are added to the lines for visibility with an interval depending on $\tau$. (d) The scaling exponents $c$, given by $\Theta \sim \tau^{c}$, for the curves of panels (a) - (c) in an intermediate time range $\tau_{\eta}<\tau \lesssim T_{L}$, with $\tau_{\eta}$ the Kolmogorov time scale and $T_{L}$ the Lagrangian integral timescale. The vertical black dashed line corresponds to Ro $=2.5$ and the solid horizontal black line is the HIT prediction $c=0.5$.

time increments $\tau$. Furthermore, especially in the regime of Ro $\lesssim 2.5$, strong peaks are observed at the end of the curves in Fig. 6(e). These peaks are related to large values of $\theta$, where the Taylor expansion of Eq. (12) is not valid anymore, explaining why here the curves deviate from the Fisher distribution.

\section{Average angle of directional change}

Next, we want to understand whether rotation affects the scaling of the average angle of directional change $\Theta(\tau)$ with $\tau$ and in particular whether a signature of the transition in the typical coherent flow structures can be found at timescales larger than the Kolmogorov time. In Figs. 7(a) and 7(b), the average angle $\Theta(\tau)$ is shown as a function of $\tau$, for different rotation rates in the center and near the top plate of the RBC cell. For small values of $\tau$ a ballistic regime is recovered where $\Theta(\tau) \sim \tau$ for all rotation rates. A second scaling range can be identified for $\tau_{\eta}<\tau \lesssim T_{L}$, where a transition has occurred from the ballistic regime to a different, lower, scaling exponent. This scaling exponent at intermediate timescales is moreover decreasing with rotation, a trend we will discuss in more detail later. Note that in the non-BL region and in the regime of Ro $\gtrsim 2.5$ [Fig. 7(b)], curves of $\Theta$ extend up to lower values of $\tau$ compared to the center [Fig. 7(a)]. This is a result of the smaller measurement volume used near the top plate, compared to the center, resulting in shorter trajectories.

Inside the viscous BL, two clear regimes are distinguished in Fig. 7 (c) with a transition around Ro $\approx 2.5$, in contrast to what we observed in the bulk. In the regime of the LSC, where Ro $\gtrsim$ 2.5 , curves deflect downwards for large $\tau .^{2}$ This is related to the strong horizontal mean flow present in the Prandtl-Blasius $\mathrm{BL}$, giving rise to lower average angles along the trajectories. When Ro $\lesssim 2.5$, the boundary layer is of the Ekman type and there is interaction between the BL and the bulk, also known as Ekman pumping or suction $[9,29,41]$. This might explain why here the $\Theta(\tau)$ vs $\tau$ curve is again similar to that measured in the non-BL [Fig. 7(b)] and in the center [Fig. 7(a)].

To quantify the change in the scaling exponent under rotation in Figs. 7(a)-7(c), a linear fit of $\ln (\Theta(\tau))$ as a function of $\ln (\tau)$ is performed in the range of $\tau_{\eta}<\tau<T_{L}$, measuring

\footnotetext{
${ }^{2}$ Note that for Ro $=2.5$, where the transition from the LSC to vertically aligned plumes occurs, the downward deflection of $\Theta(\tau)$ for large times is less prominent in Fig. 7(c), due to the breakdown of the LSC at this Rossby number.
} 
the scaling exponent $c$ as $\Theta(\tau) \sim \tau^{c}$. Computing the scaling exponent $c$ is very sensitive to the definition of the fitting range and therefore the error is computed as the deviation between exponents computed in five different fitting ranges: two shifted towards lower and two shifted towards higher timescales compared to the original fitting range. In Fig. 7(d), $c$ is shown as a function of Ro for DNS, showing all three measurement volumes. Both in the center and the non-BL region two regimes can be distinguished: in the regime of the LSC (Ro $\gtrsim 2.5$ ) the scaling exponent is a constant: $c=0.66 \pm 0.03$ in the center and $c=0.49 \pm 0.06$ near the top plate, while in the regime of vertically aligned plumes (Ro $\lesssim 2.5) c$ is decreasing with decreasing Ro and approaching $c=0.19 \pm 0.04$ (for the highest rotation rates in our simulations). Inside the BL, exponents are lower compared to the bulk and the deviation between the bulk and BL measurements are especially large in the regime where the LSC is the dominant flow structure.

\section{Effect of large-scale coherent flow structures on the angle of directional change}

In (nonrotating) HIT, $\Theta(\tau)$ is expected to scale as $\tau^{1 / 2}$, when $\tau_{\eta}<\tau<T_{L}$, based on Kolmogorov phenomenology. Since the level of turbulence studied here is moderate $\left(\operatorname{Re}_{\lambda} \approx 39\right.$ for $\operatorname{Ro}=\infty$ to $\operatorname{Re}_{\lambda} \approx 7$ for $\operatorname{Ro}=0.05$ ), we do not expect the energy spectrum to show a clear inertial range and it is therefore not obvious whether we can apply these arguments, used in HIT, to explain the scaling exponents measured in the RBC flow. Instead, we focus on the influence of the confinement and of the dominant flow structures in RBC, which are the LSC for Ro $\gtrsim 2.5$ and the vertically aligned plumes for Ro $\lesssim 2.5$, and investigate how they affect the scaling exponent in the range of $\tau_{\eta}<\tau<T_{L}$.

A first factor that is expected to affect the scaling exponents is the confinement, induced by the finite measurement volume. In smaller measurement volumes, trajectories have to be more "curved" in order to fit inside, while in larger measurement volumes also longer, more stretched, trajectories are allowed. This will result in smaller average angles at larger time increments, for larger measurement volumes. We define the size of the measurement volume relative to the total volume of the RBC cell as $V / V_{\text {tot }}$, where $V_{\text {tot }}=\pi / 4$ and $V=\Delta x \times \Delta y \times \Delta z$ with $\Delta_{i}$ the size of the measurement volume in the $i$ th direction (in dimensionless units). It is important to distinguish the effect of the confinement from the effect of the typical flow structures on the exponents $c$. Therefore, we show $c$ as a function of Ro for the original central measurement volume of $V / V_{\text {tot }}=0.16$ and an enlarged measurement volume of $V / V_{\text {tot }}=0.65$ in Fig. 8(a). Values of $c$ are lower for $V / V_{\text {tot }}=0.65$, exactly for the reason mentioned above; smaller measurement volumes will result in more "curved" trajectories and therefore larger values of $\Theta$ and larger values of $c$. However, the trend of constant $c$ for Ro $\gtrsim 2.5$ and decreasing $c$ with decreasing Ro for Ro $\lesssim 2.5$ is still clearly visible, indicating that this is not an effect of confinement but probably related to the coherent flow structures. Moreover, for Ro $\gtrsim 2.5$, exponents are still larger than the HIT value of $c=0.5$.

For Ro $\gtrsim 2.5$ the LSC is expected to induce a vertical shear flow upon trajectories in the center, expressed by an up-going motion on one side of the measurement volume, and

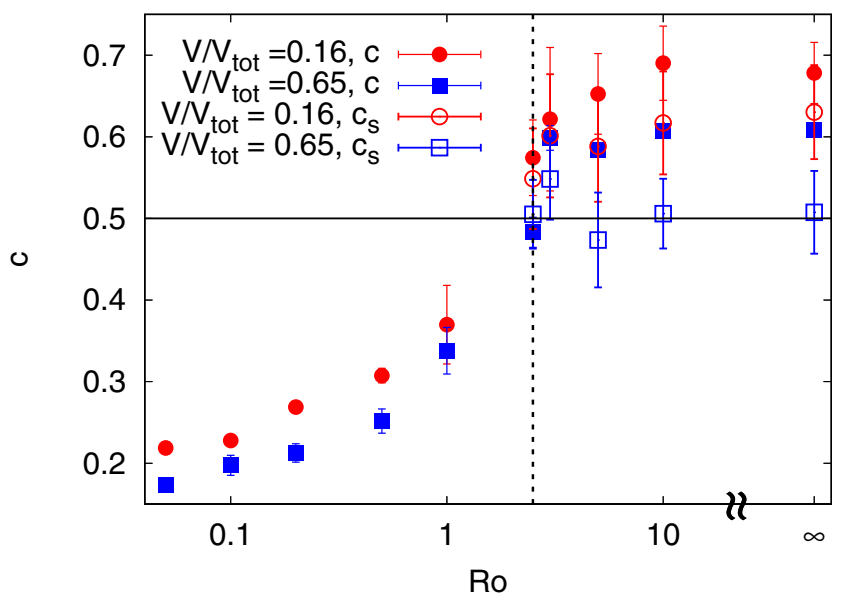

(a) shear correction

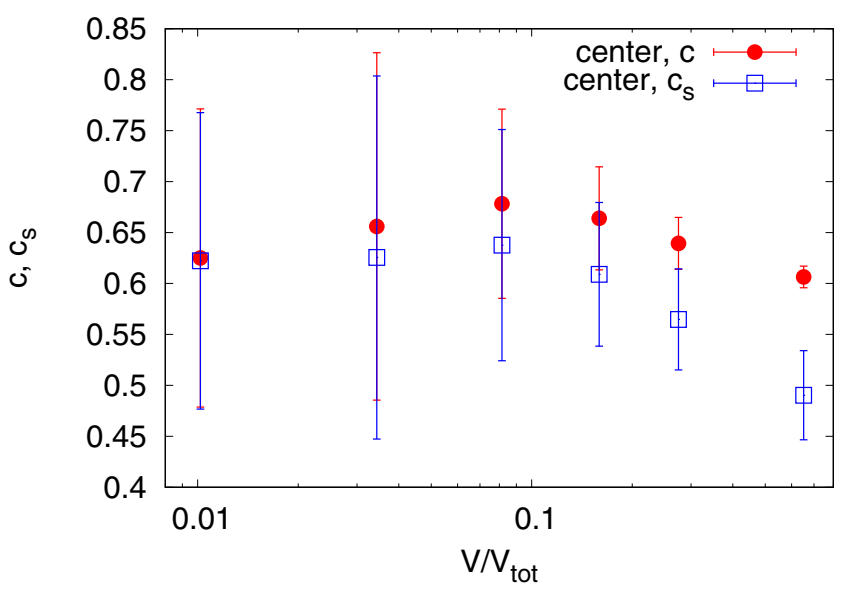

(b) measurement volume

FIG. 8. (a) Scaling exponents without and with shear correction ( $c$ and $c_{s}$, open and closed symbols, respectively), measured in the center of the Rayleigh-Bénard cell for $\tau_{\eta}<\tau \lesssim T_{L}$, with $\tau_{\eta}$ the Kolmogorov timescale and $T_{L}$ the Lagrangian integral timescale. Red circles correspond to a relative volume of size $V / V_{\text {tot }}=0.16$ and blue squares correspond to $V / V_{\text {tot }}=0.65$, where $V=\Delta x \times \Delta y \times \Delta z$ with $\Delta_{i}$ the size of the measurement volume in the $i$ th direction. The vertical black dashed line corresponds to Ro $=2.5$ and the solid horizontal black line is the HIT prediction $c=0.5$. (b) $c$ and $c_{s}$ for Ro $=\infty$ as a function of $V / V_{\text {tot }}$. The measurement volume is always centered in the middle of the RBC cell and has the same size in all directions, such that $\Delta x=\Delta y=\Delta z$.

a down-going motion on the other side. Near the top plate, the LSC already induces a mean horizontal flow, giving rise to straighter trajectories and lower angles and therefore lower values of $c$ in Fig. 7(d). To correct for the LSC induced shear flow in the center, we first compute the average vertical (shear) flow, $U_{s}(x, y)$, by binning the measurement volume in the $x$ and $y$ directions and computing the average vertical velocity in each bin, averaged over time and over the $z$ direction. Then, the displacement caused by $U_{s}(x, y)$ is subtracted from the trajectories and the average angle is computed for these corrected trajectories as a function of $\tau$, referred to as $\Theta_{s}(\tau)$. From $\Theta_{s}(\tau)$ we compute the scaling exponent $c_{s}$ as $\Theta_{s}(\tau) \sim$ $\tau^{c_{s}}$ in the regime $\tau_{\eta}<\tau<T_{L}$. The strength of the shear 
flow, induced by the LSC, is expected to depend on $V / V_{\text {tot }}$ since the closer the measurement volume is to the walls, the more the LSC will penetrate the volume. To understand this, we compute both $\Theta_{s}(\tau)$ and $\Theta(\tau)$ (without shear correction) in different measurement volumes $V / V_{\text {tot }}$, for Ro $=\infty$. In Fig. 8(b), $c$ and $c_{s}$ are shown to decrease with increasing $V / V_{\text {tot }}$ when $V / V_{\text {tot }} \gtrsim 0.08$ and shear correction is decreasing the exponent further where the difference between $c$ and $c_{s}$ is larger for larger $V / V_{\text {tot }}$. Indeed, for larger measurement volumes the LSC is expected to be more dominant and shear correction more important. Now, we moreover observe that $c_{s}$ decreases up to $c \approx 0.49 \pm 0.04$, so to the HIT scaling exponent, for $V / V_{\text {tot }}=0.65$. We now include $c_{s}$ measured for $V / V_{\text {tot }}=0.65$ for the other Rossby numbers in the regime of the LSC (Ro $\gtrsim 2.5$ ) in Fig. 8(a). These exponents are now actually identical to the HIT prediction, suggesting that, in the center, it is indeed the vertical shear induced by the LSC and the confinement of the measurement volume causing the exponents to be larger than the HIT prediction.

In the weakly rotation-affected regime, where Ro $\lesssim 2.5$, the scaling exponent is found to decrease with decreasing Ro, or increasing rotation rate. To understand this trend, we consider the coherence time of the dominant large-scale flow structures in the two rotational regimes. In particular, the vertical coherence of the vortical structures is larger than that of the LSC, as also evident from Table I. This implies that trajectories are correlated for a longer time when Ro $\lesssim 2.5$. Considering that the convergence to $2 \pi / 3$ is reached when the three subsequent points along the trajectories (see sketch in Fig. 1) are randomly placed in the (confined) domain, it is expected that this convergence is reached at a later time for Ro $\lesssim 2.5$ as confirmed in Figs. 7(a) and 7(b). In these figures we also observe that, just before reaching the convergence to $2 \pi / 3, \Theta(\tau)$ is lower for Ro $\lesssim 2.5$, compared to Ro $\gtrsim 2.5$, in the center. To understand this, we consider the example of spiral-like trajectories, which are much more common in the weakly rotation-affected regime, compared to the rotation-unaffected regime. Such a spiral-shaped trajectory is characterized by circular motions, resulting in a "return" point each time a circle is completed. This is expected to have two main effects: (i) For $\tau<\tau_{\eta}$, these circular motions are expected to increase the angle of directional change, explaining why $\Theta(\tau)$ is larger for Ro $\lesssim 2.5$ in the ballistic regime in Figs. 7(a) and 7(b). (ii) For $\tau_{\eta}<\tau<T_{L}$, the spiral-like trajectories reveal lower angles of directional change due to the returning points. This suggests that for Ro $\lesssim 2.5$ lower angles are measured even for larger $\tau$. We verify above arguments by computing $P_{\theta}$ for a low time increment, $\tau=0.1$, and a large time increment, $\tau=10$. Indeed, in Fig. 9, for small $\tau$ there is a higher probability on larger angles for Ro $\lesssim 2.5$ and, oppositely, for larger $\tau$ there is a higher probability on lower angles when Ro $\lesssim 2.5$, compared to Ro $\gtrsim 2.5$. All together, in the rotation-affected regime, the longer convergence time in combination with an enhanced probability on lower angles at larger $\tau$ is expected to cause the decrease in the scaling exponent with increasing rotation rate found in Fig. 7(d). Inside the BL the scaling exponents deviate from those measured in the bulk, especially in the regime dominated by the LSC. This deviation is expected to be a result of the Prandtl-Blasius BL being passive and not interacting with the bulk flow. In

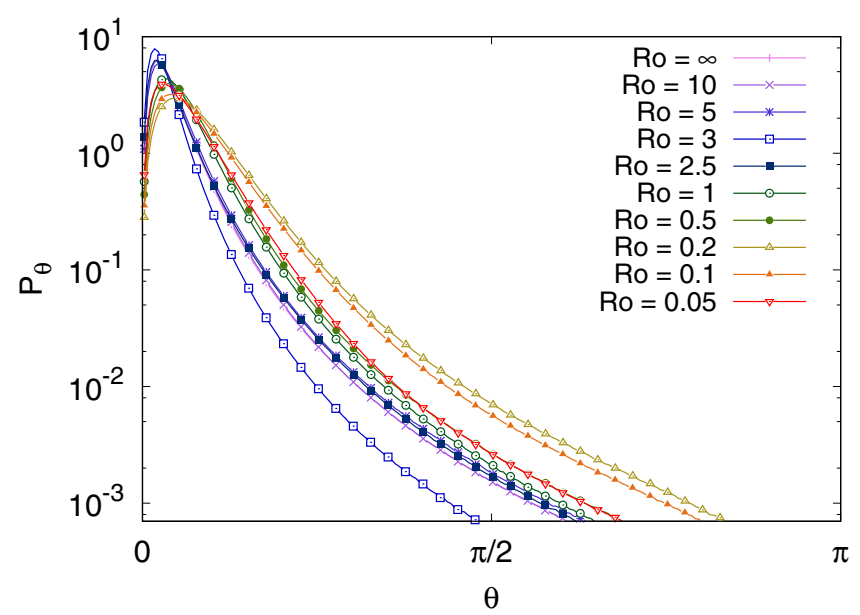

(a) $\tau=0.1$

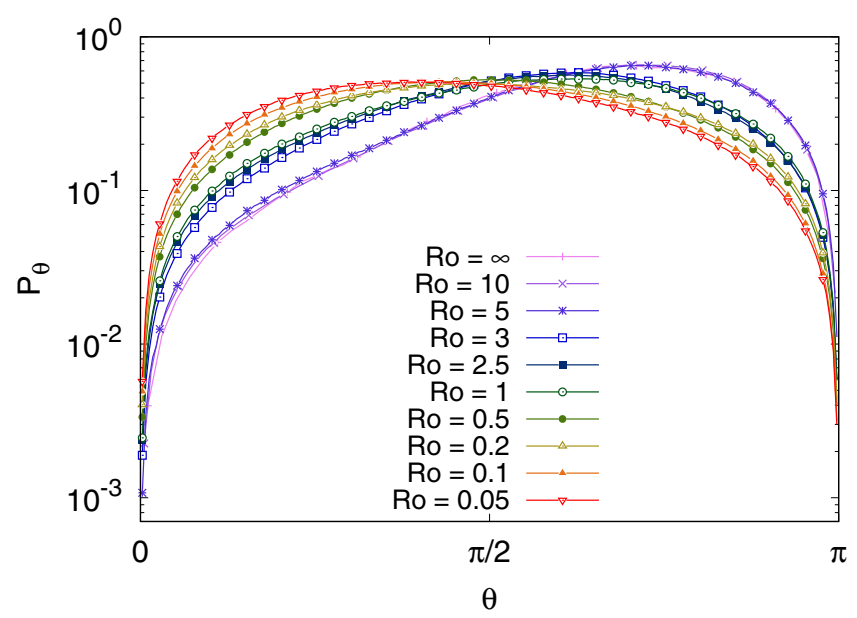

(b) $\tau=10$

FIG. 9. PDFs of the angle of directional change $\theta, P_{\theta}$, for different Rossby numbers and for a time increment of (a) $\tau=0.1$ and (b) $\tau=10$, measured numerically in the center of the Rayleigh-Bénard cell. For clarity, symbols are plotted each 5 data points.

particular, the scaling exponents are found to be lower than those measured in the bulk in this range of rotation rates. This is related to the strong mean horizontal flow present in the Prandtl-Blasius BL, decreasing $\Theta(\tau)$ even for larger timescales as also evident from Fig. 7(c). For Ro $\lesssim 2.5$, the BL is of the Ekman type and is actively interacting with the bulk flow, causing the scaling exponents to be closer to those measured in the bulk in this rotational regime.

\section{CONCLUSIONS}

The geometry of tracer trajectories is measured in (rotating) $\mathrm{RBC}$ at different timescales, as the angle of directional change. The focus is on the multiscale character of this angle and in particular it is studied how the angular statistics are affected by background rotation and coherent flow structures.

For both nonrotating and rotating RBC geometrical statistics, measured in the bulk, show a similar trend as found in HIT. Like in HIT, PDFs of $1-\cos \theta$ collapse on a Fisher 
distribution $F_{2,3}$ when normalized by $\Theta^{2}(\tau) / 3$ with $\Theta(\tau)$ the average angle of directional change. This collapse is found for all rotation rates and for time increments up to the Lagrangian integral timescale, as long as the BL is excluded. It was already found that for the instantaneous measures for the geometry of tracer trajectories, curvature, and torsion, HIT predictions are representative for RBC. Here, we study the multiscale character of this geometry and find that the HIT predictions are very robust and work even for longer timescales up to the timescale of the large-scale coherent structures, where anisotropy and inhomogeneity definitely play a role in the weakly turbulent RBC flow studied here.

Inside the viscous $\mathrm{BL}$, statistics of the angle of directional change reveal a different behavior. For small values of $\tau$, PDFs of $1-\cos \theta$ in the Ekman BL recover a Fisher distribution $F_{2,3}$, while PDFs of $1-\cos \theta$ in the Prandtl-Blasius BL approach a Fisher distribution $F_{1,2}$. This is expected to be related to the strong mean horizontal flow present in the PrandtlBlasius BL for weakly rotating RBC, effectively reducing the dimensionality of the flow and therefore the number of active independent components for the (normal) acceleration and the velocity at lower time increments $\tau$.

Previous studies have shown that, at timescales larger than the Kolmogorov timescale but smaller than the Lagrangian integral timescale, $\Theta(\tau)$ scales as $\tau^{1 / 2}$, which can be explained by invoking Kolmogorov phenomenology for the inertial range. In the RBC flow studied here, the level of turbulence is moderate (i.e., no clear inertial range develops) and the scaling in this intermediate range of timescales is not expected to display scaling in line with Kolmogorov phenomenology. Indeed, we do not see the $\Theta \sim \tau^{1 / 2}$ scaling, however, a transition is observed from the ballistic regime at small timescales, where $\Theta \sim \tau$, to a scaling with a lower exponent at intermediate timescales. This scaling exponent is found to be constant for Ro $\gtrsim 2.5$ and to decrease with increasing rotation rate for Ro $\lesssim 2.5$. In the center of the RBC cell, the constant scaling is larger than the HIT prediction. In this regime of rotation rates, the dominant flow structure is the LSC, inducing a vertical shear flow upon the trajectories. It is found that this shear flow, together with the confinement of the measurement volume, is causing the scaling exponent to be larger than the HIT prediction. When the measurement volume is large enough (more than $65 \%$ of the total cell size) and trajectories are corrected for the shear flow, the HIT scaling is actually recovered. In the weakly rotation-affected regime, the dominant flow structures are vertically aligned vortices. Now, the range of timescales in which the scaling can be identified is extended due to the longer vertical coherence of these vertically aligned plumes. Furthermore, the presence of more spiral-like trajectories is resulting in lower average angles of directional change at larger time increments. This is resulting in a decrease of the scaling exponent with increasing rotation rate when Ro $\lesssim 2.5$. Again, different behavior is found in the viscous BLs at the plates; in the passive Prandtl-Blasius BL exponents are much lower than those measured in the bulk, while in the Ekman BL, that actively interacts with the bulk, exponents are closer to those measured in the bulk.

So, the scaling of the average angle $\Theta(\tau)$ with $\tau$ provides useful information on the coherent flow structures in rotating $\mathrm{RBC}$ at different timescales and both in the bulk and in the BL. To distinguish between the effect of the coherent flow structures and the typical characteristics of turbulence, such as the Kolmogorov scaling of the energy spectrum, simulations at larger Rayleigh numbers should be performed in future research. A wider inertial range is expected to develop allowing for a further comparison to the results of HIT. Furthermore, the coherent structures are expected to change again at even lower Rossby numbers; here, the vertically aligned vortices start to form columnar structures of the size of the cell height. The transition to this third regime is much less explored since it requires a high level of turbulence in a rotationally constrained flow. Since the scaling of the average angle with time has shown to provide useful information on the coherent flow structures, this transition can be studied in more detail by extending the range of Rossby numbers studied here to lower values in future work.

\section{ACKNOWLEDGMENTS}

This work is financially supported by the Nederlandse Organisatie voor Wetenschappelijk Onderzoek I (NWO-I), the Netherlands. The authors gratefully acknowledge the support of NWO for the use of supercomputer facilities (Cartesius) under Grant No. 16289. EU-COST action MP1305 "Flowing matter" is kindly acknowledged. The authors thank K. Schneider and W. Bos for their valuable input on this work.
[1] J.-C. Gascard, A. J. Watson, M.-J. Messias, K. A. Olsson, T. Johannessen, and K. Simonsen, Nature (London) 416, 525 (2002).

[2] J. Marshall and F. Schott, Rev. Geophys. 37, 1 (1999).

[3] M. S. Miesch, Solar Phys. 192, 59 (2000).

[4] M. G. Dunn, J. Turbomach. 123, 637 (2001).

[5] J. P. Johnston, Int. J. Rotating Mach. 4, 97 (1998).

[6] E. J. Hopfinger, F. K. Browand, and Y. Gagne, J. Fluid Mech. 125, 505 (1982).

[7] L. J. A. van Bokhoven, H. J. H. Clercx, G. J. F. van Heijst, and R. R. Trieling, Phys. Fluids 21, 096601 (2009).

[8] R. P. J. Kunnen, H. J. H. Clercx, and B. J. Geurts, Europhys. Lett. 84, 24001 (2008).
[9] R. J. A. M. Stevens, J.-Q. Zhong, H. J. H. Clercx, G. Ahlers, and D. Lohse, Phys. Rev. Lett. 103, 024503 (2009).

[10] P. Wei, S. Weiss, and G. Ahlers, Phys. Rev. Lett. 114, 114506 (2015).

[11] H. Rajaei, P. Joshi, K. M. J. Alards, R. P. J. Kunnen, F. Toschi, and H. J. H. Clercx, Phys. Rev. E 93, 043129 (2016).

[12] W. Braun, F. De Lillo, and B. Eckhardt, J. Turbul. 7, N62 (2006).

[13] Y. Choi, Y. Park, and C. Lee, Int. J. Heat Fluid Flow 31, 482 (2010).

[14] A. Scagliarini, J. Turbul. 12, N25 (2011).

[15] H. Xu, N. T. Ouellette, and E. Bodenschatz, Phys. Rev. Lett. 98, 050201 (2007). 
[16] K. M. J. Alards, H. Rajaei, L. Del Castello, R. P. J. Kunnen, F. Toschi, and H. J. H. Clercx, Phys. Rev. Fluids 2, 044601 (2017).

[17] W. J. T. Bos, B. Kadoch, and K. Schneider, Phys. Rev. Lett. 114, 214502 (2015).

[18] B. Kadoch, M. Bassenne, M. Esmaily-Moghadam, K. Schneider, M. Farge, and W. J. T. Bos, in Proceedings of the Summer Program (Center for Turbulence Research, California, 2016), https://ctr.stanford.edu/proceedings-2016-summer-program.

[19] B. Kadoch, W. J. T. Bos, and K. Schneider, Phys. Rev. Fluids 2, 064604 (2017).

[20] R. P. J. Kunnen, B. J. Geurts, and H. J. H. Clercx, J. Fluid Mech. 642, 445 (2010).

[21] R. P. J. Kunnen, H. J. H. Clercx, and B. J. Geurts, Phys. Rev. Lett. 101, 174501 (2008).

[22] H. Rajaei, P. Joshi, R. P. J. Kunnen, and H. J. H. Clercx, Phys. Rev. Fluids 1, 044403 (2016).

[23] D. Lohse and K.-Q. Xia, Annu. Rev. Fluid Mech. 42, 335 (2010).

[24] H. Rajaei, K. M. J. Alards, R. P. J. Kunnen, and H. J. H. Clercx (unpublished).

[25] O. Zeman, Phys. Fluids 6, 3221 (1994).

[26] Y. Zhou, Phys. Fluids 7, 2092 (1995).

[27] F. S. Godeferd and F. Moisy, Appl. Mech. Rev. 67, 030802 (2015).

[28] E. M. King, S. Stellmach, J. Noir, U. Hansen, and J. M. Aurnou, Nature (London) 457, 301 (2009).
[29] K. Julien, S. Legg, J. McWilliams, and J. Werne, Phys. Rev. E 53, R5557 (1996).

[30] S. Burov, S. M. A. Tabei, T. Huynh, M. P. Murrell, L. H. Philipson, S. A. Rice, M. L. Gardel, N. F. Scherer, and A. R. Dinner, Proc. Natl. Acad. Sci. U. S. A. 110, 19689 (2013).

[31] L. Biferale, G. Boffetta, A. Celani, B. J. Devenish, A. Lanotte, and F. Toschi, Phys. Rev. Lett. 93, 064502 (2004).

[32] F. Moisy and J. Jiménez, J. Fluid Mech. 513, 111 (2004).

[33] S. B. Pope, Turbulent Flows (Cambridge University Press, New York, 2000).

[34] R. Verzicco and P. Orlandi, J. Comput. Phys. 123, 402 (1996).

[35] R. Verzicco and R. Camussi, J. Fluid Mech. 477, 19 (2003).

[36] B. Lüthi, Some aspects of strain, vorticity and material element dynamics as measured with 3D particle tracking velocimetry in a turbulent flow, Ph.D. thesis, Swiss Federal Institue of Technology, Switzerland, 2002.

[37] J. Willneff, Int. Arch. Photogramm. Rem. Sens. Spatial Inform. Sci. 34, 601 (2002).

[38] Q. Zhou, C. Sun, and K.-Q. Xia, J. Fluid Mech. 598, 361 (2008).

[39] O. Shishkina, S. Horn, and S. Wagner, J. Fluid Mech. 730, 442 (2013).

[40] D. R. Caldwell and C. W. V. Atta, J. Fluid Mech. 44, 79 (1970).

[41] H. T. Rossby, J. Fluid Mech. 36, 309 (1969). 